\title{
Convergence to common solutions of various problems for nonexpansive mappings in Hilbert spaces
}

Kyung Soo Kim*

\author{
"Correspondence: \\ kksmj@kyungnam.ac.kr \\ Graduate School of Education, \\ Mathematics Education, Kyungnam \\ University, Changwon, Kyungnam \\ 631-701, Republic of Korea
}

\begin{abstract}
In this paper, motivated and inspired by Ceng and Yao (J. Comput. Appl. Math. 214(1):186-201, 2008), liduka and Takahashi (Nonlinear Anal. 61(3):341-350, 2005), Jaiboon and Kumam (Nonlinear Anal. 73(5):1180-1202, 2010), Kim (Nonlinear Anal. 73:3413-3419, 2010), Marino and Xu (J. Math. Anal. Appl. 318:43-52, 2006) and Saeidi (Nonlinear Anal. 70:4195-4208, 2009), we introduce a new iterative scheme for finding a common element of the set of solutions of a mixed equilibrium problem for an equilibrium bifunction, the set of fixed points of an infinite family of nonexpansive mappings, the set of solutions of some variational inequality problem, and the set of fixed points of a left amenable semigroup $\left\{T_{t}: t \in S\right\}$ of nonexpansive mappings with respect to $W$-mappings and a left regular sequence $\left\{\mu_{n}\right\}$ of means defined on an appropriate space of bounded real-valued functions of the semigroup $S$. Furthermore, we prove that the iterative scheme converges strongly to a common element of the above four sets. Our results extend and improve the corresponding results of many others.

MSC: 43A65; 47H05; 47H09; 47H10; 47J20; 47J25; 74G40
\end{abstract}

Keywords: mixed equilibrium problem; variational inequality problem; reversible semigroup; mean; common fixed point

\section{Introduction}

Let $H$ be a real Hilbert space, let $C$ be a nonempty closed convex subset of $H$, and let $P_{C}$ be the metric projection of $H$ onto $C$. Let $\varphi: C \rightarrow \mathbb{R}$ be a real-valued function and $\theta: C \times C \rightarrow \mathbb{R}$ be an equilibrium bifunction with $\theta(u, u)=0$ for each $u \in C$. We consider the mixed equilibrium problem (for short, $M E P$ ) is to find $x^{*} \in C$ such that

$$
M E P: \theta\left(x^{*}, y\right)+\varphi(y)-\varphi\left(x^{*}\right) \geq 0, \quad \forall y \in C .
$$

In particular, if $\varphi \equiv 0$, this problem reduces to the equilibrium problem (for short, $E P$ ), which is to find $x^{*} \in C$ such that

$$
E P: \theta\left(x^{*}, y\right) \geq 0, \quad \forall y \in C .
$$

Denote the set of solutions of $M E P$ by $\Omega$. The mixed equilibrium problems include fixed point problems, optimization problems, variational inequality problems, Nash equilibrium problems and the equilibrium problems as special cases.

(c) 2012 Kim; licensee Springer. This is an Open Access article distributed under the terms of the Creative Commons Attribution License (http://creativecommons.org/licenses/by/2.0), which permits unrestricted use, distribution, and reproduction in any medium, provided the original work is properly cited. 
A mapping $T$ of $C$ into itself is called nonexpansive if

$$
\|T x-T y\| \leq\|x-y\|
$$

for all $x, y \in C$. We denote by $F(T)$ the set of fixed points of $T$. It is well known that $F(T)$ is closed convex. Recall that a mapping $f: C \rightarrow C$ is called contractive if there exists a constant $\alpha \in(0,1)$ such that

$$
\|f(x)-f(y)\| \leq \alpha\|x-y\|
$$

for all $x, y \in C$.

In 2000, Moudafi [1] introduced the viscosity approximation method for nonexpansive mappings (see [2] for further developments in both Hilbert and Banach spaces).

Starting with an arbitrary initial $x_{0} \in H$, define a sequence $\left\{x_{n}\right\}$ recursively by

$$
x_{n+1}=\left(1-\alpha_{n}\right) T x_{n}+\alpha_{n} f\left(x_{n}\right), \quad n \geq 0,
$$

where $\alpha_{n}$ is a sequence in $(0,1)$. It is proved that under certain appropriate conditions imposed on $\left\{\alpha_{n}\right\}$, the sequence $\left\{x_{n}\right\}$ generated by (1.1) strongly converges to the unique solution $x^{\prime \prime}$ in $F(T)$ of the variational inequality

$$
\left\langle(f-I) x^{*}, x-x^{*}\right\rangle \leq 0, \quad \forall x \in F(T)
$$

(see $[1,2])$.

Let $A$ be a strongly positive bounded linear operator on $H$, that is, there exists a constant $\bar{\gamma}>0$ such that

$$
\langle A x, x\rangle \geq \bar{\gamma}\|x\|^{2},
$$

for all $x \in H$.

In 2006, Marino and Xu [3] considered the following iterative method:

$$
x_{n+1}=\left(I-\alpha_{n} A\right) T x_{n}+\alpha_{n} \gamma f\left(x_{n}\right), \quad n \geq 0,
$$

where $0<\gamma<\frac{\bar{\gamma}}{\alpha}, \alpha$ is a contraction coefficient of $f$. They proved that if the sequence $\left\{\alpha_{n}\right\}$ satisfies appropriate conditions, then the sequence $\left\{x_{n}\right\}$ generated by (1.2) converges strongly to the unique solution of the variational inequality

$$
\left\langle(A-\gamma f) x^{*}, x-x^{*}\right\rangle \geq 0, \quad x \in F(T)
$$

which is the optimality condition for the minimization problem

$$
\min _{x \in F(T)} \frac{1}{2}\langle A x, x\rangle-h(x)
$$

where $h$ is a potential function for $\gamma f\left(\right.$ i.e., $h^{\prime}(x)=\gamma f(x)$, for $x \in H$ ). 
A set-valued mapping $T: H \rightarrow 2^{H}$ is called monotone if for all $x, y \in H, f \in T x$ and $g \in T y$ imply $\langle x-y, f-g\rangle \geq 0$. A monotone mapping $T: H \rightarrow 2^{H}$ is maximal if its graph $G(T)$ is not properly contained in the graph of any other monotone mapping. It is known that a monotone mapping $T$ is maximal if and only if for $(x, f) \in H \times H,\langle x-y, f-g\rangle \geq 0$ for every $(y, g) \in G(T)$ implies $f \in T x$. Let $A$ be a monotone mapping of $C$ into $H$, and let $N_{C} v$ be the normal cone to $C$ at $v \in C$, i.e.,

$$
N_{C} v=\{\mathrm{w} \in H:\langle v-u, \mathrm{w}\rangle \geq 0, \forall u \in C\}
$$

and define

$$
T v= \begin{cases}A v+N_{C} v, & \text { if } v \in C, \\ \emptyset, & \text { if } v \notin C .\end{cases}
$$

Then $T$ is maximal monotone, and $0 \in T v$ if and only if $v \in V I(C, A)$; see [4].

In 2005, for finding an element of $F(T) \cap V I(C, A)$, Iiduka and Takahashi [5] proposed a new iterative sequence: $x_{1}=x \in C$ and

$$
x_{n+1}=\alpha_{n} x+\left(1-\alpha_{n}\right) T P_{C}\left(x_{n}-\lambda_{n} A x_{n}\right), \quad n \geq 1
$$

and obtained a strong convergence theorem in a Hilbert space.

Let $\left\{T_{n}\right\}$ be a sequence of nonexpansive mappings of $C$ into itself, and let $\left\{\lambda_{n}\right\}$ be a sequence of nonnegative numbers in $[0,1]$. For each $n \geq 1$, define a mapping $W_{n}$ of $C$ into itself as follows:

$$
\begin{aligned}
& U_{n, n+1}=I \text {, } \\
& U_{n, n}=\lambda_{n} T_{n} U_{n, n+1}+\left(1-\lambda_{n}\right) I, \\
& U_{n, n-1}=\lambda_{n-1} T_{n-1} U_{n, n}+\left(1-\lambda_{n-1}\right) I, \\
& U_{n, k}=\lambda_{k} T_{k} U_{n, k+1}+\left(1-\lambda_{k}\right) I, \\
& U_{n, k-1}=\lambda_{k-1} T_{k-1} U_{n, k}+\left(1-\lambda_{k-1}\right) I \text {, } \\
& U_{n, 2}=\lambda_{2} T_{2} U_{n, 3}+\left(1-\lambda_{2}\right) I \text {, } \\
& W_{n}=U_{n, 1}=\lambda_{1} T_{1} U_{n, 2}+\left(1-\lambda_{1}\right) I .
\end{aligned}
$$

Such a mapping $W_{n}$ is called the $W$-mapping generated by $T_{1}, T_{2}, \ldots, T_{n}$ and $\lambda_{1}, \lambda_{2}, \ldots, \lambda_{n}$. The concept of $W$-mapping was introduced in [6,7] and [8].

In 2008, Ceng and Yao [9] introduced the hybrid iterative scheme

$$
\left\{\begin{array}{l}
x_{0} \in C \quad \text { arbitrary, } \\
\theta\left(y_{n}, x\right)+\varphi(x)-\varphi\left(y_{n}\right)+\frac{1}{r}\left\langle K^{\prime}\left(y_{n}\right)-K^{\prime}\left(x_{n}\right), \eta\left(x, y_{n}\right)\right\rangle \geq 0, \quad \forall x \in C, \\
x_{n+1}=\alpha_{n} f\left(W_{n} x_{n}\right)+\beta_{n} x_{n}+\gamma_{n} W_{n} y_{n},
\end{array}\right.
$$


where $K^{\prime}(x)$ is the Fréchet derivative of $K$ at $x$. They proved the sequences $\left\{x_{n}\right\}$ and $\left\{y_{n}\right\}$ generated by the hybrid iterative scheme (1.5) converge strongly to a common element of the set of solutions of $M E P$ and the set of common fixed points of finitely many nonexpansive mappings.

Recall the mapping $B$ is said to be relaxed $(\xi, v)$-cocoercive, if there exist two constants $\xi, v>0$ such that

$$
\langle B x-B y, x-y\rangle \geq-\xi\|B x-B y\|^{2}+v\|x-y\|^{2}, \quad \forall x, y \in C .
$$

This class of mappings has been considered by many authors; for example, [10, 11].

In this paper, motivated and inspired by Ceng and Yao [9], Iiduka and Takahashi [5], Jaiboon and Kumam [12], Kim [13], Marino and Xu [3] and Saeidi [14], we introduce a new iterative scheme:

$$
\left\{\begin{array}{l}
x_{0} \in C \quad \text { arbitrary, } \\
\theta\left(z_{n}, x\right)+\varphi(x)-\varphi\left(z_{n}\right)+\frac{1}{r_{n}}\left\langle K^{\prime}\left(z_{n}\right)-K^{\prime}\left(x_{n}\right), \eta\left(x, z_{n}\right)\right\rangle \geq 0, \\
y_{n}=\left(1-\gamma_{n}\right) x_{n}+\gamma_{n} T_{\mu_{n}} W_{n} P_{C}\left(I-\delta_{n} B\right) z_{n}, \\
x_{n+1}=\alpha_{n} \gamma f\left(W_{n} x_{n}\right)+\beta_{n} x_{n}+\left(\left(1-\beta_{n}\right) I-\alpha_{n} A\right) T_{\mu_{n}} W_{n} y_{n},
\end{array}\right.
$$

for all $x \in C, n \geq 0$, for finding a common element of the set of solutions of a mixed equilibrium problem for an equilibrium bifunction, the set of fixed points of an infinite family of nonexpansive mappings, the set of solutions of some variational inequality problem and the set of fixed points of a left amenable semigroup $\left\{T_{t}: t \in S\right\}$ of nonexpansive mappings with respect to $W$-mappings and a left regular sequence $\left\{\mu_{n}\right\}$ of means defined on an appropriate space of bounded real-valued functions of the semigroup $S$. Furthermore, we prove that the proposed iterative scheme (1.6) converges strongly to a common element of the above four sets. Our result extends and improves the corresponding results of many others.

\section{Preliminaries}

Let $S$ be a semigroup. We denote by $B(S)$ the space of all bounded real-valued functions defined on $S$ with supremum norm. For each $s \in S$, we define the left and right translation operators $l_{s}$ and $r_{s}$ on $B(S)$ by

$$
\left(l_{s} f\right)(t)=f(s t) \quad \text { and } \quad\left(r_{s} f\right)(t)=f(t s)
$$

for each $t \in S$ and $f \in B(S)$, respectively. Let $X$ be a subspace of $B(S)$ containing 1 . An element $\mu$ in the dual space $X^{*}$ of $X$ is said to be a mean on $X$ if $\|\mu\|=\mu(1)=1$. For $s \in S$, we can define a point evaluation $\delta_{s}$ by $\delta_{s}(f)=f(s)$ for each $f \in X$. It is well known that $\mu$ is a mean on $X$ if and only if

$$
\inf _{s \in S} f(s) \leq \mu(f) \leq \sup _{s \in S} f(s)
$$

for each $f \in X$. 
Let $X$ be a translation invariant subspace of $B(S)$ (i.e., $l_{s} X \subset X$ and $r_{s} X \subset X$ for each $s \in S$ ) containing 1 . Then a mean $\mu$ on $X$ is said to be left invariant (resp. right invariant) if

$$
\mu\left(l_{s} f\right)=\mu(f) \quad\left(\text { resp. } \mu\left(r_{s} f\right)=\mu(f)\right)
$$

for each $s \in S$ and $f \in X$. A mean $\mu$ on $X$ is said to be invariant if $\mu$ is both left and right invariant [15-17]. $X$ is said to be left (resp. right) amenable if $X$ has a left (resp. right) invariant mean. $X$ is amenable if $X$ is left and right amenable. Moreover, $B(S)$ is amenable when $S$ is a commutative semigroup or a solvable group. However, the free group or semigroup of two generators is not left or right amenable. In this case, we say that the semigroup $S$ is an amenable semigroup (see $[18,19])$. A semigroup $S$ is left reversible if $S$ has the finite intersection property for right ideals. Every left reversible semigroup $S, W A P(S)$ the space of weakly almost period functions on $S$ has a left invariant mean. If $S$ is both left and right reversible, then $W A P(S)$ has an invariant mean. Each group or amenable semigroup is left and right reversible (see $[20,21])$.

A net $\left\{\mu_{\alpha}\right\}$ of means on $X$ is said to be asymptotically left (resp. right) invariant if

$$
\lim _{\alpha}\left(\mu_{\alpha}\left(l_{s} f\right)-\mu_{\alpha}(f)\right)=0 \quad\left(\text { resp. } \lim _{\alpha}\left(\mu_{\alpha}\left(r_{s} f\right)-\mu_{\alpha}(f)\right)=0\right)
$$

for each $f \in X$ and $s \in S$, and it is said to be left (resp. right) strongly asymptotically invariant (or strong regular) if

$$
\lim _{\alpha}\left\|l_{s}^{*} \mu_{\alpha}-\mu_{\alpha}\right\|=0 \quad\left(\text { resp. } \lim _{\alpha}\left\|r_{s}^{*} \mu_{\alpha}-\mu_{\alpha}\right\|=0\right)
$$

for each $s \in S$, where $l_{s}^{*}$ and $r_{s}^{*}$ are the adjoint operators of $l_{s}$ and $r_{s}$, respectively. Such nets were first studied by Day in [18] where they were called weak" invariant and norm invariant, respectively.

It is easy to see that if a semigroup $S$ is left (resp. right) amenable, then the semigroup $S^{\prime}=S \cup\{e\}$, where $e s^{\prime}=s^{\prime} e=s^{\prime}$ for all $s^{\prime} \in S$, is also left (resp. right) amenable and conversely.

Let $S$ be a semigroup, and let $C$ be a closed and convex subset of $H$. Let $F(T)$ denote the fixed point set of $T$. Then $\mathfrak{I}=\left\{T_{s}: s \in S\right\}$ is called a representation of $S$ as nonexpansive mappings on $C$ if $T_{s}$ is nonexpansive with $T_{e}=I$ and $T_{s t}=T_{s} T_{t}$ for each $s, t \in S$ (cf. [22-30]). We denote by $F(\Im)$ the set of common fixed points of $\left\{T_{s}: s \in S\right\}$, i.e.,

$$
F(\Im)=\bigcap_{s \in S} F\left(T_{s}\right)=\bigcap_{s \in S}\left\{x \in C: T_{s} x=x\right\} .
$$

Lemma 2.1 $([19,31])$ Let $S$ be a semigroup and $C$ be a closed convex subset of a Hilbert space $H$. Let $\Im=\left\{T_{s}: s \in S\right\}$ be a nonexpansive semigroup on $C$ such that $\left\{T_{t} u: t \in S\right\}$ is bounded for some $u \in C$, let $X$ be a subspace of $B(S)$ such that $1 \in X$ and the mapping $t \mapsto\left\langle T_{t} x, y\right\rangle$ is an element of $X$ for each $x \in C$ and $y \in H$, and $\mu$ be a mean on $X$. If we write $T_{\mu} x$ instead of $\int T_{t} x d \mu(t)$, then the following hold:

(i) $T_{\mu}$ is a nonexpansive mapping from $C$ into itself,

(ii) $T_{\mu} x=x$ for each $x \in F(\Im)$,

(iii) $T_{\mu} x \in \overline{\operatorname{co}}\left\{T_{t} x: t \in S\right\}$ for each $x \in C$, where $\overline{\mathrm{co}} A$ is the closed convex hull of $A$. 
Let $C$ be a nonempty subset of a Hilbert space $H$ and $T: C \rightarrow H$ be a mapping. Then $T$ is said to be demiclosed at $v \in H$ if, for any sequence $\left\{x_{n}\right\}$ in $C$, the following implication holds:

$$
x_{n} \rightarrow u \in C \text { and } T x_{n} \rightarrow v \text { imply } T u=v \text {, }
$$

where $\rightarrow$ (resp. $\rightarrow$ ) denotes strong (resp. weak) convergence.

Lemma 2.2 ([32]) Let $C$ be a nonempty closed convex subset of a Hilbert space $H$ and suppose that $T: C \rightarrow H$ is nonexpansive. Then, the mapping $I-T$ is demiclosed at zero.

Let $C$ be a nonempty subset of a normed space $E$, and let $x \in E$. An element $y_{0} \in C$ is said to be the best approximation to $x$ if

$$
\left\|x-y_{0}\right\|=d(x, C)
$$

where $d(x, C)=\inf _{y \in C}\|x-y\|$. The number $d(x, C)$ is called the distance from $x$ to $C$. Let $H$ be a real Hilbert space with inner product $\langle\cdot, \cdot\rangle$ and norm $\|\cdot\|$. Let $C$ be a nonempty closed convex subset of $H$. Then, for any $x \in H$, there exists a unique nearest point in $C$, denoted by $P_{C} x$, such that

$$
\left\|x-P_{C} x\right\| \leq\|x-y\|, \quad \forall y \in C .
$$

The mapping $P_{C}$ is called the metric projection of $H$ onto $C$. It is well known that $P_{C}$ is a nonexpansive mapping of $H$ onto $C$ and satisfies

$$
\left\langle x-y, P_{C} x-P_{C} y\right\rangle \geq\left\|P_{C} x-P_{C} y\right\|^{2}
$$

for every $x, y \in H$. Moreover, $P_{C} x$ is characterized by the following properties: $P_{C} x \in C$ and for all $x \in H, y \in C$,

$$
\left\langle x-P_{C} x, y-P_{C} x\right\rangle \leq 0
$$

and

$$
\|x-y\|^{2} \geq\left\|x-P_{C} x\right\|^{2}+\left\|y-P_{C} x\right\|^{2} .
$$

It is easy to see that the following is true:

$$
u \in V I(C, B) \quad \Longleftrightarrow \quad u=P_{C}(u-\lambda B u), \quad \lambda>0 .
$$

In this paper, for solving the mixed equilibrium problems for an equilibrium bifunction $\theta: C \times C \rightarrow \mathbb{R}$, we assume that $\theta$ satisfies the following conditions:

(E1) $\theta(x, x)=0$ for all $x \in C$; 
(E2) $\theta$ is monotone, i.e., $\theta(x, y)+\theta(y, x) \leq 0$ for all $x, y \in C$;

(E3) for each $x, y, z \in C$,

$$
\lim _{t \downarrow 0} \theta(t z+(1-t) x, y) \leq \theta(x, y)
$$

(E4) for each $x \in C$, the function $y \mapsto \theta(x, y)$ is convex and lower semicontinuous.

Definition 2.1 (1) Let $F: C \rightarrow H$ and $\eta: C \times C \rightarrow H$ be two mappings. Then $F$ is called:

(i) $\eta$-monotone if

$$
\langle F(x)-F(y), \eta(x, y)\rangle \geq 0, \quad \forall x, y \in C
$$

(ii) $\eta$-strongly monotone with constant $\alpha$ if there exists a constant $\alpha>0$ such that

$$
\langle F(x)-F(y), \eta(x, y)\rangle \geq \alpha\|x-y\|^{2}, \quad \forall x, y \in C,
$$

(iii) Lipschitz continuous with constant $\beta$ if there exists a constant $\beta>0$ such that

$$
\|F(x)-F(y)\| \leq \beta\|x-y\|, \quad \forall x, y \in C
$$

If $\eta(x, y)=x-y$, for all $x, y \in C$, then the definitions (i) and (ii) reduce to the definition of monotonicity and strong monotonicity, respectively.

(2) A mapping $\eta: C \times C \rightarrow H$ is called Lipschitz continuous with constant $\lambda$ if there exists a constant $\lambda>0$ such that

$$
\|\eta(x, y)\| \leq \lambda\|x-y\|, \quad \forall x, y \in C
$$

(3) A differentiable function $K: C \rightarrow \mathbb{R}$ on a convex set $C$ is called:

(i) $\eta$-convex $[33]$ if

$$
K(y)-K(x) \geq\left\langle K^{\prime}(x), \eta(y, x)\right\rangle, \quad \forall x, y \in C,
$$

where $K^{\prime}(x)$ is the Fréchet derivative of $K$ at $x$,

(ii) $\eta$-strongly convex with constant $\sigma$ [34] if there exists a constant $\sigma>0$ such that

$$
K(y)-K(x)-\left\langle K^{\prime}(x), \eta(y, x)\right\rangle \geq \frac{\sigma}{2}\|x-y\|^{2}, \quad \forall x, y \in C
$$

(4) A mapping $F: C \rightarrow \mathbb{R}$ is called sequentially continuous at $x_{0}$ [35], if $F\left(x_{n}\right) \rightarrow F\left(x_{0}\right)$ for each sequence $\left\{x_{n}\right\}$ satisfying $x_{n} \rightarrow x_{0} . F$ is called sequentially continuous on $C$ if it is sequentially continuous at each point of $C$.

Lemma 2.3 ([9]) Let $K: C \rightarrow \mathbb{R}$ be differentiable $\eta$-strongly convex with a constant $\sigma>0$, and let $\eta: C \times C \rightarrow H$ be a mapping such that $\eta(x, y)+\eta(y, x)=0$ for all $x, y \in C$. Then $K^{\prime}: C \rightarrow H$ is $\eta$-strongly monotone with constant $\sigma>0$. 
Lemma 2.4 ([36]) A Hilbert space $H$ is said to satisfy Opial's condition iffor each sequence $\left\{x_{n}\right\}$ in $H$, the condition $x_{n} \rightarrow x$ implies that

$$
\liminf _{n \rightarrow \infty}\left\|x_{n}-x\right\|<\liminf _{n \rightarrow \infty}\left\|x_{n}-y\right\|
$$

for all $y \in H$ with $y \neq x$.

Lemma 2.5 Let $H$ be a real Hilbert space. Then

$$
\|x+y\|^{2} \leq\|x\|^{2}+2\langle y, x+y\rangle,
$$

for all $x, y \in H$.

Let $C$ be a nonempty closed convex subset of a real Hilbert space $H, \varphi: C \rightarrow \mathbb{R}$ be a real-valued function and $\theta: C \times C \rightarrow \mathbb{R}$ be an equilibrium bifunction. Let $r$ be a positive parameter. For a given point $x \in C$, consider the auxiliary problem for the mixed equilibrium problem (for short, $\operatorname{MEP}(x, r)$ ) which consists of finding $y \in C$ such that

$$
\theta(y, z)+\varphi(z)-\varphi(y)+\frac{1}{r}\left\langle K^{\prime}(y)-K^{\prime}(x), \eta(z, y)\right\rangle \geq 0, \quad \forall z \in C,
$$

where $\eta: C \times C \rightarrow H$ and $K^{\prime}(x)$ is the Fréchet derivative of a functional $K: C \rightarrow \mathbb{R}$ at $x$. Let $S_{r}: C \rightarrow C$ be the mapping such that for each $x \in C, S_{r}(x)$ is the solution set of $M E P(x, r)$, i.e.,

$$
\begin{aligned}
S_{r}(x)= & \{y \in C: \theta(y, z)+\varphi(z)-\varphi(y) \\
& \left.+\frac{1}{r}\left\langle K^{\prime}(y)-K^{\prime}(x), \eta(z, y)\right\rangle \geq 0, \forall z \in C\right\}
\end{aligned}
$$

for all $x \in C$.

We first need the following important and interesting result.

Lemma 2.6 ([9]) Let $C$ be a nonempty closed convex subset of a real Hilbert space $H$, and let $\varphi: C \rightarrow \mathbb{R}$ be a lower semicontinuous and convex functional. Let $\theta: C \times C \rightarrow \mathbb{R}$ be an equilibrium bifunction satisfying conditions (E1)-(E4). Assume that

(i) $\eta: C \times C \rightarrow H$ is Lipschitz continuous with constant $\lambda>0$ such that

(a) $\eta(x, y)+\eta(y, x)=0, \forall x, y \in C$,

(b) for each fixed $y \in C, x \mapsto \eta(y, x)$ is sequentially continuous from the weak topology to the weak topology,

(ii) $K: C \rightarrow \mathbb{R}$ is $\eta$-strongly convex with constant $\sigma>0$ and its derivative $K^{\prime}$ is sequentially continuous from the weak topology to the strong topology,

(iii) for each $x \in C$, there exist a bounded subset $D_{x} \subseteq C$ and $z_{x} \in C$ such that for any $y \in C \backslash D_{x}$,

$$
\theta\left(y, z_{x}\right)+\varphi\left(z_{x}\right)-\varphi(y)+\frac{1}{r}\left\langle K^{\prime}(y)-K^{\prime}(x), \eta\left(z_{x}, y\right)\right\rangle<0 .
$$

Then the following hold:

(1) $S_{r}$ is single-valued; 
(2) $S_{r}$ is a firmly nonexpansive-type mapping, i.e., for all $x, y \in H$,

$$
\left\|S_{r} x-S_{r} y\right\|^{2} \leq\left\langle S_{r} x-S_{r} y, x-y\right\rangle
$$

(3) (j) $S_{r}$ is nonexpansive if $K^{\prime}$ is Lipschitz continuous with constant $v>0$ such that $\sigma \geq \lambda \nu$

(jj) $\left\langle K^{\prime}\left(x_{1}\right)-K^{\prime}\left(x_{2}\right), \eta\left(u_{1}, u_{2}\right)\right\rangle \geq\left\langle K^{\prime}\left(u_{1}\right)-K^{\prime}\left(u_{2}\right), \eta\left(u_{1}, u_{2}\right)\right\rangle, \forall\left(x_{1}, x_{2}\right) \in C \times C$, where $u_{i}=S_{r}\left(x_{i}\right), i=1,2$;

(4) $F\left(S_{r}\right)=\Omega$;

(5) $\Omega$ is a closed and convex subset of $C$.

Remark 2.1 In particular, from Lemma 2.6, whenever $K(x)=\frac{\|x\|^{2}}{2}$ and $\eta(x, y)=x-y$ for each $(x, y) \in C \times C$, then $S_{r}$ is firmly nonexpansive, i.e.,

$$
\left\langle x_{1}-x_{2}, S_{r}\left(x_{1}\right)-S_{r}\left(x_{2}\right)\right\rangle \geq\left\|S_{r}\left(x_{1}\right)-S_{r}\left(x_{2}\right)\right\|^{2}, \quad \forall\left(x_{1}, x_{2}\right) \in C \times C .
$$

We need the following results concerning the $W$-mapping $W_{n}$.

Lemma 2.7 ([37]) Let $C$ be a nonempty closed convex subset of a real Hilbert space H. Let $T_{1}, T_{2}, \ldots$ be nonexpansive mappings of $C$ into $H$ such that $\bigcap_{i=1}^{\infty} F\left(T_{i}\right)$ is nonempty, and let $\lambda_{1}, \lambda_{2}, \ldots$ be real numbers such that $0<\lambda_{i} \leq b<1$ for any $i \in \mathbb{N}$. Then, for every $x \in C$ and $k \in \mathbb{N}$, the limit $\lim _{n \rightarrow \infty} U_{n, k} x$ exists.

Using Lemma 2.7, one can define a mapping $W$ of $C$ into $H$ as

$$
W x=\lim _{n \rightarrow \infty} W_{n} x=\lim _{n \rightarrow \infty} U_{n, 1} x
$$

for every $x \in C$.

Remark 2.2 ([37]) Let $C$ be a nonempty closed convex subset of a real Hilbert space $H$. Let $T_{1}, T_{2}, \ldots$ be nonexpansive mappings of $C$ into $H$ such that $\bigcap_{i=1}^{\infty} F\left(T_{i}\right)$ is nonempty, and let $\lambda_{1}, \lambda_{2}, \ldots$ be real numbers such that $0<\lambda_{i} \leq b<1$ for any $i \in \mathbb{N}$. Then $F(W)=\bigcap_{i=1}^{\infty} F\left(T_{i}\right)$.

Remark 2.3 ([38]) Let $C$ be a nonempty closed convex subset of a real Hilbert space $H$. Let $T_{1}, T_{2}, \ldots$ be nonexpansive mappings of $C$ into $H$ such that $\bigcap_{i=1}^{\infty} F\left(T_{i}\right)$ is nonempty. If $\left\{x_{n}\right\}$ is an arbitrary bounded sequence in $C$, then we have

$$
\lim _{n \rightarrow \infty}\left\|W x_{n}-W_{n} x_{n}\right\|=0
$$

Lemma 2.8 ([39]) Let $\left\{x_{n}\right\}$ and $\left\{z_{n}\right\}$ be bounded sequences in a Hilbert space $H$ and let $\left\{\beta_{n}\right\}$ be a sequence in $[0,1]$ with $0<\liminf _{n \rightarrow \infty} \beta_{n}$ and $\limsup _{n \rightarrow \infty} \beta_{n}<1$. Suppose

$$
x_{n+1}=\beta_{n} x_{n}+\left(1-\beta_{n}\right) z_{n}
$$

for all integers $n \geq 0$ and

$$
\limsup _{n \rightarrow \infty}\left(\left\|z_{n+1}-z_{n}\right\|-\left\|x_{n+1}-x_{n}\right\|\right) \leq 0 .
$$

Then $\lim _{n \rightarrow \infty}\left\|z_{n}-x_{n}\right\|=0$. 
Lemma 2.9 ([3]) Assume $A$ is a strongly positive linear bounded operator on a Hilbert space $H$ with a coefficient $\bar{\gamma}>0$ and $0<\rho \leq\|A\|^{-1}$. Then $\|I-\rho A\| \leq 1-\rho \bar{\gamma}$.

Lemma 2.10 ([2]) Assume $\left\{a_{n}\right\}$ is a sequence of nonnegative real numbers such that

$$
a_{n+1} \leq\left(1-b_{n}\right) a_{n}+b_{n} c_{n}
$$

where $\left\{b_{n}\right\}$ is a sequence in $(0,1)$ and $\left\{c_{n}\right\}$ is a sequence in $\mathbb{R}$ such that

(1) $\sum_{n=1}^{\infty} b_{n}=\infty$,

(2) $\lim \sup _{n \rightarrow \infty} c_{n} \leq 0$ or $\sum_{n=1}^{\infty}\left|b_{n} c_{n}\right|<\infty$.

Then

$$
\lim _{n \rightarrow \infty} a_{n}=0 .
$$

\section{Main result: strong convergence theorems}

In this section, we deal with the strong convergence of hybrid viscosity approximation scheme (1.6) for finding a common element of the set of solutions of a mixed equilibrium problem, the set of fixed points of an infinite family of nonexpansive mappings, the set of fixed points of a left amenable semigroup of nonexpansive mappings and the set of solutions of variational inequality in a Hilbert space.

Theorem 3.1 Let $S$ be a semigroup, $\Im=\left\{T_{t}: t \in S\right\}$ be a nonexpansive semigroup on $H$ such that $F(\Im) \neq \emptyset, X$ be a left invariant subspace of $B(S)$ such that $1 \in X$, and the function $t \mapsto\left\langle T_{t} x, y\right\rangle$ is an element of $X$ for each $x, y \in H$. Let $\left\{\mu_{n}\right\}$ be a left strong regular sequence of means on $X$ such that $\lim _{n \rightarrow \infty}\left\|\mu_{n+1}-\mu_{n}\right\|=0$. Let $C$ be a nonempty closed convex subset of a real Hilbert space $H$ and $\left\{T_{i}\right\}$ be an infinite family of nonexpansive mappings from $C$ into itself such that $T_{i}(F(\Im) \cap \Omega) \subset F(\Im)$ for each $i \in \mathbb{N}$. Let $\varphi: C \rightarrow \mathbb{R}$ be a lower semicontinuous and convex functional. Let $\theta: C \times C \rightarrow \mathbb{R}$ be an equilibrium bifunction satisfying conditions (E1)-(E4), and let $T_{1}, T_{2}, \ldots$ be an infinite family of nonexpansive mappings of $C$ into $H$. Let $r>0, \gamma>0$ be two constants. Let $f$ be a contraction of $C$ into itself with a coefficient $\alpha \in(0,1)$, and let $A$ be a strongly positive bounded linear operator with a coeffcient $\bar{\gamma}>0$ such that $0<\alpha \gamma<\bar{\gamma}<\alpha \gamma+1$. Let $B: C \rightarrow H$ be an L-Lipschitzian and relaxed $(\xi, \nu)$-cocoercive mapping. Suppose that $\mathcal{F}=\bigcap_{n=1}^{\infty} F\left(T_{n}\right) \cap F(\Im) \cap \Omega \cap V I(C, B) \neq \emptyset$. Let $\left\{\alpha_{n}\right\}$, $\left\{\beta_{n}\right\}$ and $\left\{\gamma_{n}\right\}$ be sequences in $[0,1]$ such that $\alpha_{n}+\beta_{n} \leq 1$, and let the sequence $\left\{\delta_{n}\right\} \subset(0, \infty)$. Assume that:

(C1) $\eta: C \times C \rightarrow H$ is Lipschitz continuous with constant $\lambda>0$ such that

(a) $\eta(x, y)+\eta(y, x)=0, \forall x, y \in C$,

(b) for each fixed $y \in C, x \mapsto \eta(y, x)$ is sequentially continuous from the weak topology to the weak topology,

(C2) $K: C \rightarrow \mathbb{R}$ is $\eta$-strongly convex with constant $\sigma>0$ and its derivative $K^{\prime}$ is not only sequentially continuous from the weak topology to the strong topology, but also Lipschitz continuous with constant $v>0$ such that $\sigma \geq \lambda \nu$,

(C3) for each $x \in C$, there exist a bounded subset $D_{x} \subset C$ and $z_{x} \in C$ such that for any $y \in C \backslash D_{x}$,

$$
\theta\left(y, z_{x}\right)+\varphi\left(z_{x}\right)-\varphi(y)+\frac{1}{r}\left\langle K^{\prime}(y)-K^{\prime}(x), \eta\left(z_{x}, y\right)\right\rangle<0,
$$


(C4) (i) $\lim _{n \rightarrow \infty} \alpha_{n}=0, \sum_{n=0}^{\infty} \alpha_{n}=\infty$,

(ii) $0<\liminf _{n \rightarrow \infty} \beta_{n} \leq \limsup _{n \rightarrow \infty} \beta_{n}<1$,

$0<\liminf _{n \rightarrow \infty} \gamma_{n} \leq \limsup _{n \rightarrow \infty} \gamma_{n}<1$,

(iii) $\lim _{n \rightarrow \infty}\left|\delta_{n+1}-\delta_{n}\right|=0, a \leq \delta_{n} \leq b$ for some $a, b$ with $0 \leq a \leq b \leq \frac{2\left(\nu-\xi L^{2}\right)}{L^{2}}$,

(iv) $\lim _{n \rightarrow \infty}\left|\gamma_{n+1}-\gamma_{n}\right|=0$,

(C5) $\liminf _{n \rightarrow \infty} r_{n}>0$ and $\lim _{n \rightarrow \infty}\left|r_{n+1}-r_{n}\right|=0$.

Given $x_{0} \in C$ is arbitrary, then the sequences $\left\{x_{n}\right\},\left\{y_{n}\right\}$ and $\left\{z_{n}\right\}$ generated iteratively by (1.6) converge strongly to $x^{*} \in \mathcal{F}$, where $x^{*}=P_{\mathcal{F}}(\gamma f+(I-A)) x^{*}$, which solves the following variational inequality:

$$
\left\langle(\gamma f-A) x^{\prime \prime}, x-x^{\prime \prime}\right\rangle \leq 0, \quad \forall x \in \mathcal{F} .
$$

Lemma 3.1 $\left\|\left(1-\beta_{n}\right) I-\alpha_{n} A\right\| \leq 1-\beta_{n}-\alpha_{n} \bar{\gamma}$.

Proof Since $\lim _{n \rightarrow \infty} \alpha_{n}=0$, we may assume, without loss of generality, that $\alpha_{n} \leq(1-$ $\left.\beta_{n}\right)\|A\|^{-1}$. Since $A$ is a linear bounded self-adjoint operator on $H$, we have

$$
\|A\|=\sup \{|\langle A x, x\rangle|: x \in H,\|x\|=1\} .
$$

Observe that

$$
\begin{aligned}
\left\langle\left(\left(1-\beta_{n}\right) I-\alpha_{n} A\right) x, x\right\rangle & =1-\beta_{n}-\alpha_{n}\langle A x, x\rangle \\
& \geq 1-\beta_{n}-\alpha_{n}\|A\| \\
& \geq 0,
\end{aligned}
$$

which shows that $\left(1-\beta_{n}\right) I-\alpha_{n} A$ is positive. By Lemma 2.9, we have

$$
\left\|\left(1-\beta_{n}\right) I-\alpha_{n} A\right\| \leq 1-\beta_{n}-\alpha_{n} \bar{\gamma} .
$$

Lemma 3.2 Let $B$ be an L-Lipschitzian and relaxed $(\xi, \nu)$-cocoercive mapping and $\delta_{n} \leq$ $\frac{2\left(v-\xi L^{2}\right)}{L^{2}}$, then

$$
\left\|\left(I-\delta_{n} B\right) x-\left(I-\delta_{n} B\right) y\right\| \leq\|x-y\|,
$$

for all $x, y \in C$.

Proof Since $B$ is an $L$-Lipschitzian and relaxed $(\xi, v)$-cocoercive mapping and $\delta_{n} \leq$ $\frac{2\left(v-\xi L^{2}\right)}{L^{2}}$, we have

$$
\begin{aligned}
\| & \left(I-\delta_{n} B\right) x-\left(I-\delta_{n} B\right) y \|^{2} \\
& =\|x-y\|^{2}-2 \delta_{n}\langle B x-B y, x-y\rangle+\delta_{n}^{2}\|B x-B y\|^{2} \\
& \leq\|x-y\|^{2}-2 \delta_{n}\left(-\xi\|B x-B y\|^{2}+v\|x-y\|^{2}\right)+\delta_{n}^{2} L^{2}\|x-y\|^{2} \\
& \leq\|x-y\|^{2}+2 \delta_{n} \xi L^{2}\|x-y\|^{2}-2 \delta_{n} v\|x-y\|^{2}+\delta_{n}^{2} L^{2}\|x-y\|^{2} \\
& =\left(1+2 \delta_{n} \xi L^{2}-2 \delta_{n} v+\delta_{n}^{2} L^{2}\right)\|x-y\|^{2} \leq\|x-y\|^{2},
\end{aligned}
$$


for all $x, y \in C$. Thus,

$$
\left\|\left(I-\delta_{n} B\right) x-\left(I-\delta_{n} B\right) y\right\| \leq\|x-y\|,
$$

for all $x, y \in C$.

Lemma 3.3 $\left\|z_{n}-p\right\| \leq\left\|x_{n}-p\right\|, \forall p \in \mathcal{F}$.

Proof From (2.3), we note that $z_{n}=S_{r_{n}} x_{n}$. From Lemma 2.6, we get

$$
\left\|z_{n}-p\right\| \leq\left\|S_{r_{n}} x_{n}-S_{r_{n}} p\right\| \leq\left\|x_{n}-p\right\|
$$

for all $p \in \mathcal{F}$.

Lemma 3.4 $\left\{x_{n}\right\},\left\{y_{n}\right\},\left\{z_{n}\right\},\left\{W_{n} y_{n}\right\},\left\{W_{n} x_{n}\right\}$ and $\left\{f\left(W_{n} x_{n}\right)\right\}$ are all bounded.

Proof Let $p \in \mathcal{F}$. Since $p \in V I(C, B)$, from (2.2), we get $p=P_{C}\left(I-\delta_{n} B\right) p$. From Lemma 2.1, Lemma 3.3 and $W_{n}, P_{C}$ being nonexpansive, we have

$$
\begin{aligned}
\left\|y_{n}-p\right\| & =\left\|\left(1-\gamma_{n}\right) x_{n}+\gamma_{n} T_{\mu_{n}} W_{n} P_{C}\left(I-\delta_{n} B\right) z_{n}-p\right\| \\
& \leq\left(1-\gamma_{n}\right)\left\|x_{n}-p\right\|+\gamma_{n}\left\|T_{\mu_{n}} W_{n} P_{C}\left(I-\delta_{n} B\right) z_{n}-p\right\| \\
& \leq\left(1-\gamma_{n}\right)\left\|x_{n}-p\right\|+\gamma_{n}\left\|\left(I-\delta_{n} B\right) z_{n}-\left(I-\delta_{n} B\right) p\right\| \\
& \leq\left(1-\gamma_{n}\right)\left\|x_{n}-p\right\|+\gamma_{n}\left\|z_{n}-p\right\| \\
& \leq\left\|x_{n}-p\right\| .
\end{aligned}
$$

From (1.6) and Lemma 3.1, we obtain

$$
\begin{aligned}
&\left\|x_{n+1}-p\right\| \\
&=\left\|\alpha_{n} \gamma f\left(W_{n} x_{n}\right)+\beta_{n} x_{n}+\left(\left(1-\beta_{n}\right) I-\alpha_{n} A\right) T_{\mu_{n}} W_{n} y_{n}-p\right\| \\
&= \| \alpha_{n}\left(\gamma f\left(W_{n} x_{n}\right)-A p\right)+\beta_{n}\left(x_{n}-p\right) \\
&+\left(\left(1-\beta_{n}\right) I-\alpha_{n} A\right)\left(T_{\mu_{n}} W_{n} y_{n}-p\right) \| \\
& \leq \alpha_{n}\left\|\gamma f\left(W_{n} x_{n}\right)-A p\right\|+\beta_{n}\left\|x_{n}-p\right\|+\left\|\left(1-\beta_{n}\right) I-\alpha_{n} A\right\|\left\|y_{n}-p\right\| \\
& \leq\left(1-\beta_{n}-\alpha_{n} \bar{\gamma}\right)\left\|y_{n}-p\right\|+\beta_{n}\left\|x_{n}-p\right\| \\
&+\alpha_{n} \gamma\left\|f\left(W_{n} x_{n}\right)-f(p)\right\|+\alpha_{n}\|\gamma f(p)-A p\| \\
& \leq\left(1-\alpha_{n} \bar{\gamma}\right)\left\|x_{n}-p\right\|+\alpha_{n} \gamma \alpha\left\|x_{n}-p\right\|+\alpha_{n}\|\gamma f(p)-A p\| \\
&=\left(1-\alpha_{n}(\bar{\gamma}-\gamma \alpha)\right)\left\|x_{n}-p\right\|+\alpha_{n}(\bar{\gamma}-\gamma \alpha) \frac{\|\gamma f(p)-A p\|}{\bar{\gamma}-\gamma \alpha},
\end{aligned}
$$

for all $n \geq 0$. It follows by mathematical induction that

$$
\left\|x_{n+1}-p\right\| \leq \max \left\{\left\|x_{0}-p\right\|, \frac{\|\gamma f(p)-A p\|}{\bar{\gamma}-\gamma \alpha}\right\}, \quad n \geq 0 .
$$

Therefore, $\left\{x_{n}\right\}$ is bounded. We also deduce that $\left\{y_{n}\right\},\left\{z_{n}\right\},\left\{W_{n} y_{n}\right\},\left\{W_{n} x_{n}\right\}$ and $\left\{f\left(W_{n} x_{n}\right)\right\}$ are all bounded. 
Lemma 3.5 Let the mapping $W_{n}$ be generated iteratively by (1.5). If $\left\{\omega_{n}\right\}$ is a bounded sequence in $H$, then

(1) $\lim _{n \rightarrow \infty}\left\|W_{n+1} \omega_{n}-W_{n} \omega_{n}\right\|=0$.

(2) $\lim _{n \rightarrow \infty}\left\|T_{\mu_{n+1}} \omega_{n}-T_{\mu_{n}} \omega_{n}\right\|=0$.

Proof (1) We shall use $M$ to denote the possible different constants appearing in the following argument. From (1.5), since $T_{i}$ and $U_{n, i}$ are nonexpansive, we have

$$
\begin{aligned}
\left\|W_{n+1} \omega_{n}-W_{n} \omega_{n}\right\| & =\left\|\lambda_{1} T_{1} U_{n+1,2} \omega_{n}+\left(1-\lambda_{1}\right) \omega_{n}-\lambda_{1} T_{1} U_{n, 2} \omega_{n}-\left(1-\lambda_{1}\right) \omega_{n}\right\| \\
& \leq \lambda_{1}\left\|U_{n+1,2} \omega_{n}-U_{n, 2} \omega_{n}\right\| \\
& =\lambda_{1}\left\|\lambda_{2} T_{2} U_{n+1,3} \omega_{n}+\left(1-\lambda_{2}\right) \omega_{n}-\lambda_{2} T_{2} U_{n, 3} \omega_{n}-\left(1-\lambda_{2}\right) \omega_{n}\right\| \\
& \leq \lambda_{1} \lambda_{2}\left\|U_{n+1,3} \omega_{n}-U_{n, 3} \omega_{n}\right\| \\
& \leq \lambda_{1} \lambda_{2} \cdots \lambda_{n}\left\|U_{n+1, n+1} \omega_{n}-U_{n, n+1} \omega_{n}\right\| \\
& \leq M \prod_{i=1}^{n} \lambda_{i},
\end{aligned}
$$

which implies that

$$
\lim _{n \rightarrow \infty}\left\|W_{n+1} \omega_{n}-W_{n} \omega_{n}\right\|=0 .
$$

(2) Let $q \in F(\Im)$. Then $\left\|T_{s} \omega_{n}-q\right\| \leq\left\|\omega_{n}-q\right\|$. Also, we have

$$
\left\|T_{s} \omega_{n}\right\| \leq\left\|\omega_{n}-q\right\|+\|q\|
$$

for all $s \in S$ and $n \geq 0$. Since $\left\{\omega_{n}\right\}$ is bounded and $\lim _{n \rightarrow \infty}\left\|\mu_{n+1}-\mu_{n}\right\|=0$, we get

$$
\begin{aligned}
\| T_{\mu_{n+1} \omega_{n}-T_{\mu_{n}} \omega_{n} \|} & =\sup \left\{\left|\left\langle T_{\mu_{n+1}} \omega_{n}-T_{\mu_{n}} \omega_{n}, z\right\rangle\right|:\|z\|=1\right\} \\
& =\sup \left\{\left|\mu_{n+1}(s)\left\langle T_{s} \omega_{n}, z\right\rangle-\mu_{n}(s)\left\langle T_{s} \omega_{n}, z\right\rangle\right|:\|z\|=1\right\} \\
& \leq\left\|\mu_{n+1}-\mu_{n}\right\| \cdot \sup _{s \in S}\left\|T_{s} \omega_{n}\right\| \\
& \leq\left\|\mu_{n+1}-\mu_{n}\right\|\left(\left\|\omega_{n}-q\right\|+\|q\|\right) \\
& \longrightarrow 0
\end{aligned}
$$

Lemma 3.6 $\lim _{n \rightarrow \infty}\left\|x_{n+1}-x_{n}\right\|=\lim _{n \rightarrow \infty}\left\|z_{n+1}-z_{n}\right\|=0$.

Proof Define a sequence $\left\{u_{n}\right\}$ by

$$
x_{n+1}=\beta_{n} x_{n}+\left(1-\beta_{n}\right) u_{n}
$$

for all $n \geq 0$. Observe that from the definition of $u_{n}$, we get

$$
\begin{aligned}
u_{n+1}-u_{n} & =\frac{x_{n+2}-\beta_{n+1} x_{n+1}}{1-\beta_{n+1}}-\frac{x_{n+1}-\beta_{n} x_{n}}{1-\beta_{n}} \\
& =\frac{\alpha_{n+1} \gamma f\left(W_{n+1} x_{n+1}\right)+\left(\left(1-\beta_{n+1}\right) I-\alpha_{n+1} A\right) T_{\mu_{n+1}} W_{n+1} y_{n+1}}{1-\beta_{n+1}}
\end{aligned}
$$




$$
\begin{aligned}
& -\frac{\alpha_{n} \gamma f\left(W_{n} x_{n}\right)+\left(\left(1-\beta_{n}\right) I-\alpha_{n} A\right) T_{\mu_{n}} W_{n} y_{n}}{1-\beta_{n}} \\
= & \frac{\alpha_{n+1}}{1-\beta_{n+1}} \gamma f\left(W_{n+1} x_{n+1}\right)-\frac{\alpha_{n}}{1-\beta_{n}} \gamma f\left(W_{n} x_{n}\right)+T_{\mu_{n+1}} W_{n+1} y_{n+1} \\
& -T_{\mu_{n}} W_{n} y_{n}+\frac{\alpha_{n}}{1-\beta_{n}} A T_{\mu_{n}} W_{n} y_{n}-\frac{\alpha_{n+1}}{1-\beta_{n}} A T_{\mu_{n+1}} W_{n+1} y_{n+1} \\
= & \frac{\alpha_{n+1}}{1-\beta_{n+1}}\left(\gamma f\left(W_{n+1} x_{n+1}\right)-A T_{\mu_{n+1}} W_{n+1} y_{n+1}\right) \\
& +\frac{\alpha_{n}}{1-\beta_{n}}\left(A T_{\mu_{n}} W_{n} y_{n}-\gamma f\left(W_{n} x_{n}\right)\right)+T_{\mu_{n+1}} W_{n+1} y_{n+1} \\
& -T_{\mu_{n+1}} W_{n+1} y_{n}+T_{\mu_{n+1}} W_{n+1} y_{n}-T_{\mu_{n}} W_{n} y_{n} .
\end{aligned}
$$

From (2.3), we note that $z_{n}=S_{r_{n}} x_{n}$ and $z_{n+1}=S_{r_{n+1}} x_{n+1}$, we have

$$
\begin{aligned}
& \theta\left(z_{n}, x\right)+\varphi(x)-\varphi\left(z_{n}\right)+\frac{1}{r_{n}}\left\langle K^{\prime}\left(z_{n}\right)-K^{\prime}\left(x_{n}\right), \eta\left(x, z_{n}\right)\right\rangle \geq 0 \\
& \theta\left(z_{n+1}, x\right)+\varphi(x)-\varphi\left(z_{n+1}\right)+\frac{1}{r_{n+1}}\left\langle K^{\prime}\left(z_{n+1}\right)-K^{\prime}\left(x_{n+1}\right), \eta\left(x, z_{n+1}\right)\right\rangle \geq 0
\end{aligned}
$$

for all $x \in C$. Putting $x=z_{n+1}$ in (3.3) and $x=z_{n}$ in (3.4), we have

$$
\begin{aligned}
& \theta\left(z_{n}, z_{n+1}\right)+\varphi\left(z_{n}+1\right)-\varphi\left(z_{n}\right)+\frac{1}{r_{n}}\left\langle K^{\prime}\left(z_{n}\right)-K^{\prime}\left(x_{n}\right), \eta\left(z_{n+1}, z_{n}\right)\right\rangle \geq 0, \\
& \theta\left(z_{n+1}, z_{n}\right)+\varphi\left(z_{n}\right)-\varphi\left(z_{n+1}\right)+\frac{1}{r_{n+1}}\left\langle K^{\prime}\left(z_{n+1}\right)-K^{\prime}\left(x_{n+1}\right), \eta\left(z_{n}, z_{n+1}\right)\right\rangle \geq 0 .
\end{aligned}
$$

After multiplying (3.5) and (3.6) by $r_{n}$ and adding them together, we obtain

$$
\left\langle\eta\left(z_{n+1}, z_{n}\right), K^{\prime}\left(z_{n}\right)-K^{\prime}\left(x_{n}\right)-\frac{r_{n}}{r_{n+1}}\left(K^{\prime}\left(z_{n+1}\right)-K^{\prime}\left(x_{n+1}\right)\right)\right\rangle \geq 0 .
$$

Hence,

$$
\begin{gathered}
\left\langle\eta\left(z_{n+1}, z_{n}\right), K^{\prime}\left(z_{n}\right)-K^{\prime}\left(z_{n+1}\right)+K^{\prime}\left(x_{n+1}\right)-K^{\prime}\left(x_{n}\right)\right. \\
\left.+\left(1-\frac{r_{n}}{r_{n+1}}\right)\left(K^{\prime}\left(z_{n+1}\right)-K^{\prime}\left(x_{n+1}\right)\right)\right\rangle \geq 0
\end{gathered}
$$

Then, by Lemma 2.3, we have

$$
\begin{aligned}
& \left\langle\eta\left(z_{n+1}, z_{n}\right), K^{\prime}\left(x_{n+1}\right)-K^{\prime}\left(x_{n}\right)+\left(1-\frac{r_{n}}{r_{n+1}}\right)\left(K^{\prime}\left(z_{n+1}\right)-K^{\prime}\left(x_{n+1}\right)\right)\right\rangle \\
& \quad \geq\left\langle\eta\left(z_{n}, z_{n+1}\right), K^{\prime}\left(z_{n}\right)-K^{\prime}\left(z_{n+1}\right)\right\rangle \geq \sigma\left\|z_{n}-z_{n+1}\right\|^{2},
\end{aligned}
$$

and hence

$$
\begin{aligned}
& \sigma\left\|z_{n+1}-z_{n}\right\|^{2} \\
& \quad \leq\left\|\eta\left(z_{n+1}, z_{n}\right)\right\|\left(\left\|K^{\prime}\left(x_{n+1}\right)-K^{\prime}\left(x_{n}\right)\right\|+\left(1-\frac{r_{n}}{r_{n+1}}\right)\left\|K^{\prime}\left(z_{n+1}\right)-K^{\prime}\left(x_{n+1}\right)\right\|\right) \\
& \quad \leq \lambda\left\|z_{n+1}-z_{n}\right\|\left(v\left\|x_{n+1}-x_{n}\right\|+\left(1-\frac{r_{n}}{r_{n+1}}\right) v\left\|z_{n+1}-x_{n+1}\right\|\right) .
\end{aligned}
$$


Without loss of generality, we assume that there exists a real number $k$ such that $r_{n}>k>0$ for all $n \geq 0$, we have

$$
\begin{aligned}
\left\|z_{n+1}-z_{n}\right\| & \leq \frac{\lambda v}{\sigma}\left\|x_{n+1}-x_{n}\right\|+\frac{v}{\sigma} \frac{1}{k}\left|r_{n+1}-r_{n}\right|\left\|z_{n+1}-x_{n+1}\right\| \\
& \leq\left\|x_{n+1}-x_{n}\right\|+\frac{v}{\sigma k}\left|r_{n+1}-r_{n}\right| M^{\prime}
\end{aligned}
$$

where $M^{\prime}=\sup \left\{\left\|z_{n}-x_{n}\right\|: n \geq 0\right\}$.

Setting $v_{n}=P_{C}\left(I-\delta_{n} B\right) z_{n}$ for all $n \geq 0$, from Lemma 3.2, we have

$$
\begin{aligned}
\left\|v_{n+1}-v_{n}\right\| & \leq\left\|P_{C}\left(I-\delta_{n+1} B\right) z_{n+1}-P_{C}\left(I-\delta_{n} B\right) z_{n}\right\| \\
& \leq\left\|\left(I-\delta_{n+1} B\right) z_{n+1}-\left(I-\delta_{n} B\right) z_{n}\right\| \\
& \leq\left\|\left(I-\delta_{n+1} B\right) z_{n+1}-\left(I-\delta_{n+1} B\right) z_{n}\right\|+\left\|\left(\delta_{n+1}-\delta_{n}\right) B z_{n}\right\| \\
& \leq\left\|z_{n+1}-z_{n}\right\|+\left|\delta_{n+1}-\delta_{n}\right|\left\|B z_{n}\right\| .
\end{aligned}
$$

From (3.7), we get

$$
\begin{aligned}
\| & T_{\mu_{n+1}} W_{n+1} v_{n+1}-T_{\mu_{n}} W_{n} v_{n} \| \\
\leq & \left\|T_{\mu_{n+1}} W_{n+1} v_{n+1}-T_{\mu_{n+1}} W_{n+1} v_{n}\right\|+\left\|T_{\mu_{n+1}} W_{n+1} v_{n}-T_{\mu_{n+1}} W_{n} v_{n}\right\| \\
& \quad+\left\|T_{\mu_{n+1}} W_{n} v_{n}-T_{\mu_{n}} W_{n} v_{n}\right\| \\
\leq & \left\|v_{n+1}-v_{n}\right\|+\left\|W_{n+1} v_{n}-W_{n} v_{n}\right\|+\left\|T_{\mu_{n+1}} W_{n} v_{n}-T_{\mu_{n}} W_{n} v_{n}\right\| \\
\leq & \left\|z_{n+1}-z_{n}\right\|+\left|\delta_{n+1}-\delta_{n}\right|\left\|B z_{n}\right\| \\
& \quad+\left\|W_{n+1} v_{n}-W_{n} v_{n}\right\|+\left\|T_{\mu_{n+1}} W_{n} v_{n}-T_{\mu_{n}} W_{n} v_{n}\right\| \\
\leq & \left\|x_{n+1}-x_{n}\right\|+\frac{v}{\sigma k}\left|r_{n+1}-r_{n}\right| M^{\prime}+\left|\delta_{n+1}-\delta_{n}\right|\left\|B z_{n}\right\| \\
& +\left\|W_{n+1} v_{n}-W_{n} v_{n}\right\|+\left\|T_{\mu_{n+1}} W_{n} v_{n}-T_{\mu_{n}} W_{n} v_{n}\right\| .
\end{aligned}
$$

Also, we have

$$
\begin{aligned}
&\left\|y_{n+1}-y_{n}\right\| \\
&=\left\|\left(1-\gamma_{n+1}\right) x_{n+1}+\gamma_{n+1} T_{\mu_{n+1}} W_{n+1} v_{n+1}-\left(1-\gamma_{n}\right) x_{n}-\gamma_{n} T_{\mu_{n}} W_{n} v_{n}\right\| \\
&=\|\left(1-\gamma_{n+1}\right)\left(x_{n+1}-x_{n}\right)+\left(\gamma_{n}-\gamma_{n+1}\right) x_{n} \\
& \quad+\left(\gamma_{n+1}-\gamma_{n}\right) T_{\mu_{n}} W_{n} v_{n}+\gamma_{n}\left(T_{\mu_{n+1}} W_{n+1} v_{n+1}-T_{\mu_{n}} W_{n} v_{n}\right) \| \\
& \leq\left(1-\gamma_{n+1}\right)\left\|x_{n+1}-x_{n}\right\|+\left|\gamma_{n}-\gamma_{n+1}\right|\left\|x_{n}\right\| \\
&+\left|\gamma_{n+1}-\gamma_{n}\right|\left\|T_{\mu_{n}} W_{n} v_{n}\right\|+\gamma_{n+1}\left\|T_{\mu_{n+1}} W_{n+1} v_{n+1}-T_{\mu_{n}} W_{n} v_{n}\right\| .
\end{aligned}
$$

From (3.2), we obtain

$$
\begin{aligned}
& \left\|u_{n+1}-u_{n}\right\|-\left\|x_{n+1}-x_{n}\right\| \\
& \quad \leq \frac{\alpha_{n+1}}{1-\beta_{n+1}}\left(\left\|\gamma f\left(W_{n+1} x_{n+1}\right)\right\|+\left\|A T_{\mu_{n+1}} W_{n+1} y_{n+1}\right\|\right)
\end{aligned}
$$




$$
\begin{aligned}
& \quad+\frac{\alpha_{n}}{1-\beta_{n}}\left(\left\|A T_{\mu_{n}} W_{n} y_{n}\right\|+\left\|\gamma f\left(W_{n} x_{n}\right)\right\|\right) \\
& +\left\|T_{\mu_{n+1}} W_{n+1} y_{n+1}-T_{\mu_{n+1}} W_{n+1} y_{n}\right\| \\
& +\left\|T_{\mu_{n+1}} W_{n+1} y_{n}-T_{\mu_{n}} W_{n} y_{n}\right\|-\left\|x_{n+1}-x_{n}\right\| \\
& \leq \frac{\alpha_{n+1}}{1-\beta_{n+1}}\left(\left\|\gamma f\left(W_{n+1} x_{n+1}\right)\right\|+\left\|A T_{\mu_{n+1}} W_{n+1} y_{n+1}\right\|\right) \\
& +\frac{\alpha_{n}}{1-\beta_{n}}\left(\left\|A T_{\mu_{n}} W_{n} y_{n}\right\|+\left\|\gamma f\left(W_{n} x_{n}\right)\right\|\right) \\
& +\left\|y_{n+1}-y_{n}\right\|+\left\|T_{\mu_{n+1}} W_{n+1} y_{n}-T_{\mu_{n}} W_{n} y_{n}\right\|-\left\|x_{n+1}-x_{n}\right\| .
\end{aligned}
$$

Combining (3.8), (3.9) and (3.10), we obtain

$$
\begin{aligned}
&\left\|u_{n+1}-u_{n}\right\|-\left\|x_{n+1}-x_{n}\right\| \\
& \leq \frac{\alpha_{n+1}}{1-\beta_{n+1}}\left(\left\|\gamma f\left(W_{n+1} x_{n+1}\right)\right\|+\left\|A T_{\mu_{n+1}} W_{n+1} y_{n+1}\right\|\right) \\
&+\frac{\alpha_{n}}{1-\beta_{n}}\left(\left\|A T_{\mu_{n}} W_{n} y_{n}\right\|+\left\|\gamma f\left(W_{n} x_{n}\right)\right\|\right) \\
& \leq\left(1-\gamma_{n+1}\right)\left\|x_{n+1}-x_{n}\right\|+\left|\gamma_{n}-\gamma_{n+1}\right|\left\|x_{n}\right\| \\
&+\left|\gamma_{n+1}-\gamma_{n}\right|\left\|T_{\mu_{n}} W_{n} v_{n}\right\|+\gamma_{n+1}\left(\left\|x_{n+1}-x_{n}\right\|\right. \\
&+\frac{v}{\sigma k}\left|r_{n+1}-r_{n}\right| M^{\prime}+\left|\delta_{n+1}-\delta_{n}\right|\left\|B z_{n}\right\|+\left\|W_{n+1} v_{n}-W_{n} v_{n}\right\| \\
&\left.+\left\|T_{\mu_{n+1}} W_{n} v_{n}-T_{\mu_{n}} W_{n} v_{n}\right\|\right)+\left\|T_{\mu_{n+1}} W_{n+1} y_{n}-T_{\mu_{n}} W_{n+1} y_{n}\right\| \\
&+\left\|T_{\mu_{n}} W_{n+1} y_{n}-T_{\mu_{n}} W_{n} y_{n}\right\|-\left\|x_{n+1}-x_{n}\right\| .
\end{aligned}
$$

Thus, it follows from (3.11), Lemma 3.4, Lemma 3.5 and condition (C4) that

$$
\limsup _{n \rightarrow \infty}\left(\left\|u_{n+1}-u_{n}\right\|-\left\|x_{n+1}-x_{n}\right\|\right) \leq 0 .
$$

By Lemma 2.8, we get

$$
\lim _{n \rightarrow \infty}\left\|u_{n}-x_{n}\right\|=0
$$

Consequently, we have

$$
\lim _{n \rightarrow \infty}\left\|x_{n+1}-x_{n}\right\|=\lim _{n \rightarrow \infty}\left(1-\beta_{n}\right)\left\|u_{n}-x_{n}\right\|=0 .
$$

From (3.7), we get

$$
\lim _{n \rightarrow \infty}\left\|z_{n+1}-z_{n}\right\|=0 .
$$

Lemma $3.7 \lim _{n \rightarrow \infty}\left\|x_{n}-T_{t} x_{n}\right\|=0$ for all $t \in S$.

Proof Let $p \in \mathcal{F}$ and put

$$
M_{0}=\max \left\{\left\|x_{0}-p\right\|, \frac{\|\gamma f(p)-A p\|}{\bar{\gamma}-\gamma \alpha}\right\} .
$$


Set $D=\left\{y \in H:\|y-p\| \leq M_{0}\right\}$. We remark that $D$ is a bounded closed convex set, $\left\{x_{n}\right\},\left\{y_{n}\right\},\left\{z_{n}\right\} \subset D$ and it is invariant under $\Im$ and $W_{n}$ for all $n \in \mathbb{N}$. We will show that

$$
\limsup _{n \rightarrow \infty} \sup _{y \in D}\left\|T_{\mu_{n}} y-T_{t} T_{\mu_{n}} y\right\|=0,
$$

for all $t \in S$. Let $\varepsilon>0$, by [40] (Theorem 1.2), there exists $\delta>0$ such that

$$
\overline{\operatorname{co}} F_{\delta}\left(T_{t} ; D\right)+B_{\delta} \subset F_{\varepsilon}\left(T_{t} ; D\right)
$$

for all $t \in S$. By [40] (Corollary 1.1), there exists a natural number $N$ such that

$$
\left\|\frac{1}{N+1} \sum_{i=0}^{N} T_{t^{i} s} y-T_{t}\left(\frac{1}{N+1} \sum_{i=0}^{N} T_{t^{i} s} y\right)\right\| \leq \delta,
$$

for all $t, s \in S$ and $y \in D$. Since $\left\{\mu_{n}\right\}$ is left strong regular, there exists $n_{0} \in \mathbb{N}$ such that $\left\|\mu_{n}-l_{t^{i}} \mu_{n}\right\| \leq \frac{\delta}{M_{0}+\|p\|}$ for $n \geq n_{0}$ and $i=1,2, \ldots, N$. Then we have

$$
\begin{aligned}
& \sup _{y \in D}\left\|T_{\mu_{n}} y-\int \frac{1}{N+1} \sum_{i=0}^{N} T_{t^{i} s} y d \mu_{n}(s)\right\| \\
& =\sup _{y \in D\|z\|=1}\left|\left\langle T_{\mu_{n}} y, z\right\rangle-\left\langle\int \frac{1}{N+1} \sum_{i=0}^{N} T_{t^{i} s} y d \mu_{n}(s), z\right\rangle\right| \\
& =\sup _{y \in D\|z\|=1}\left|\mu_{n}(s)\left\langle T_{s} y, z\right\rangle-\mu_{n}(s)\left\langle\frac{1}{N+1} \sum_{i=0}^{N} T_{t^{i} s} y, z\right\rangle\right| \\
& =\sup _{y \in D\|z\|=1}\left|\frac{1}{N+1} \sum_{i=0}^{N} \mu_{n}(s)\left\langle T_{s} y, z\right\rangle-\frac{1}{N+1} \sum_{i=0}^{N} \mu_{n}(s)\left\langle T_{t^{i}} y, z\right\rangle\right| \\
& \leq \frac{1}{N+1} \sum_{i=0}^{N} \sup _{y \in D} \sup _{\|z\|=1}\left|\mu_{n}(s)\left\langle T_{s} t, z\right\rangle-l_{t^{i}}^{*} \mu_{n}(s)\left\langle T_{s} y, z\right\rangle\right| \\
& \leq \max _{i=0,1,2, \ldots, N} \frac{1}{N+1} \sum_{i=0}^{N}\left\|\mu_{n}-l_{t^{i}}^{*} \mu_{n}\right\|\left\|T_{s} y\right\| \\
& \leq \max _{i=0,1,2, \ldots, N}\left\|\mu_{n}-l_{t^{i}}^{*} \mu_{n}\right\|\left(M_{0}+\|p\|\right) \leq \delta,
\end{aligned}
$$

for all $n \geq n_{0}$. By Lemma 2.1, we have

$$
\int \frac{1}{N+1} \sum_{i=0}^{N} T_{t^{i} s} y d \mu_{n}(s) \in \overline{\mathrm{co}}\left\{\frac{1}{N+1} \sum_{i=0}^{N} T_{t^{i}}\left(T_{s} y\right): s \in S\right\} .
$$

It follows from (3.14)-(3.17) that

$$
\begin{aligned}
T_{\mu_{n}} y & =\int \frac{1}{N+1} \sum_{i=0}^{N} T_{t^{i} s} y d \mu_{n}(s)+\left(T_{\mu_{n}} y-\int \frac{1}{N+1} \sum_{i=0}^{N} T_{t^{i} s} y d \mu_{n}(s)\right) \\
& \in \overline{\mathrm{co}}\left\{\frac{1}{N+1} \sum_{i=0}^{N} T_{t^{i} s} y: s \in S\right\}+B_{\delta}
\end{aligned}
$$




$$
\begin{aligned}
& \subset \overline{\operatorname{co}} F_{\delta}\left(T_{t} ; D\right)+B_{\delta} \\
& \subset T_{\varepsilon}\left(T_{t} ; D\right)
\end{aligned}
$$

for all $y \in D$ and $n \geq n_{0}$. Therefore,

$$
\limsup _{n \rightarrow \infty} \sup _{y \in D}\left\|T_{t} T_{\mu_{n}} y-T_{\mu_{n}} y\right\| \leq \varepsilon
$$

Since $\varepsilon>0$ is arbitrary, we obtain (3.13). Now, let $t \in S$ and $\varepsilon>0$. Then there exists $\delta>$ 0 , which satisfies (3.14). Take $L_{0}=(\gamma \alpha+\|A\|) M_{0}+\|\gamma f(p)-A p\|$. From $\lim _{n \rightarrow \infty} \alpha_{n}=0$, (3.12) and (3.13), there exists $k \in \mathbb{N}$ such that $\alpha_{n}<\frac{\delta\left(1-\beta_{n}\right)}{2 L_{0}}, T_{\mu_{n}} y \in F_{\delta}\left(T_{t} ; D\right)$ for all $y \in D$ and $\left\|x_{n}-x_{n+1}\right\|<\frac{\delta\left(1-\beta_{n}\right)}{2 \beta_{n}}$ for all $n \geq k$. We note that

$$
\begin{aligned}
& \left\|\alpha_{n}\left(\gamma f\left(W_{n} x_{n}\right)-A T_{\mu_{n}} W_{n} y_{n}\right)\right\| \\
& \quad \leq \alpha_{n}\left(\gamma\left\|f\left(W_{n} x_{n}\right)-f(p)\right\|+\|\gamma f(p)-A p\|+\left\|A T_{\mu_{n}} W_{n} y_{n}-A p\right\|\right) \\
& \quad \leq \alpha_{n}\left(\gamma \alpha\left\|W_{n} x_{n}-p\right\|+\|\gamma f(p)-A p\|+\|A\|\left\|T_{\mu_{n}} W_{n} y_{n}-p\right\|\right) \\
& \leq \alpha_{n}\left(\gamma \alpha\left\|x_{n}-p\right\|+\|\gamma f(p)-A p\|+\|A\|\left\|y_{n}-p\right\|\right) \\
& \leq \alpha_{n}\left((\gamma \alpha+\|A\|)\left\|x_{n}-p\right\|+\|\gamma f(p)-A p\|\right) \\
& \leq \alpha_{n}\left((\gamma \alpha+\|A\|) M_{0}+\|\gamma f(p)-A p\|\right) \\
& \leq \frac{\delta\left(1-\beta_{n}\right)}{2},
\end{aligned}
$$

for all $n \geq k$. Since

$$
\begin{aligned}
& x_{n+1}-\beta_{n} x_{n+1} \\
& \quad=\left(1-\beta_{n}\right) T_{\mu_{n}} W_{n} y_{n}+\beta_{n} x_{n}+\alpha_{n}\left(\gamma f\left(W_{n} x_{n}\right)-A T_{\mu_{n}} W_{n} y_{n}\right)-\beta_{n} x_{n+1},
\end{aligned}
$$

we get

$$
\begin{aligned}
x_{n+1}= & T_{\mu_{n}} W_{n} y_{n}+\frac{\beta_{n}}{1-\beta_{n}}\left(x_{n}-x_{n+1}\right) \\
& \quad+\frac{\alpha_{n}}{1-\beta_{n}}\left(\gamma f\left(W_{n} x_{n}\right)-A T_{\mu_{n}} W_{n} y_{n}\right) \\
\in & F_{\delta}\left(T_{t} ; D\right)+B_{\frac{\delta}{2}}+B_{\frac{\delta}{2}} \\
\subset & F_{\delta}\left(T_{t} ; D\right)+B_{\delta} \\
\subset & F_{\varepsilon}\left(T_{t} ; D\right),
\end{aligned}
$$

for all $n \geq k$. This shows that

$$
\limsup _{n \rightarrow \infty}\left\|x_{n}-T_{t} x_{n}\right\| \leq \varepsilon
$$

Since $\varepsilon>0$ is arbitrary, we get $\lim _{n \rightarrow \infty}\left\|x_{n}-T_{t} x_{n}\right\|=0$.

Lemma $3.8 \lim _{n \rightarrow \infty}\left\|x_{n}-T_{\mu_{n}} W_{n} y_{n}\right\|=0$. 
Proof Since $x_{n+1}=\alpha_{n} \gamma f\left(W_{n} x_{n}\right)+\beta_{n} x_{n}+\left(\left(1-\beta_{n}\right) I-\alpha_{n} A\right) T_{\mu_{n}} W_{n} y_{n}$, we have

$$
\begin{aligned}
\left\|x_{n}-T_{\mu_{n}} W_{n} y_{n}\right\| & \leq\left\|x_{n}-x_{n+1}\right\|+\left\|x_{n+1}-T_{\mu_{n}} W_{n} y_{n}\right\| \\
& =\left\|x_{n}-x_{n+1}\right\|+\alpha_{n}\left\|\gamma f\left(W_{n} x_{n}\right)-A T_{\mu_{n}} W_{n} y_{n}\right\|+\beta_{n}\left\|x_{n}-T_{\mu_{n}} W_{n} y_{n}\right\|,
\end{aligned}
$$

that is,

$$
\left\|x_{n}-T_{\mu_{n}} W_{n} y_{n}\right\| \leq \frac{1}{1-\beta_{n}}\left\|x_{n}-x_{n+1}\right\|+\frac{\alpha_{n}}{1-\beta_{n}}\left\|\gamma f\left(W_{n} x_{n}\right)-A T_{\mu_{n}} W_{n} y_{n}\right\|
$$

It follows from condition (C4) and Lemma 3.6 that

$$
\lim _{n \rightarrow \infty}\left\|x_{n}-T_{\mu_{n}} W_{n} y_{n}\right\|=0
$$

Lemma $3.9 \lim _{n \rightarrow \infty}\left\|x_{n}-z_{n}\right\|=0$.

Proof For $p \in \mathcal{F}$, since $S_{r}$ is firmly nonexpansive, we have

$$
\begin{aligned}
\left\|z_{n}-p\right\|^{2} & =\left\|S_{r_{n}} x_{n}-S_{r_{n}} p\right\|^{2} \\
& \leq\left\langle S_{r_{n}} x_{n}-S_{r_{n}} p, x_{n}-p\right\rangle=\left\langle z_{n}-p, x_{n}-p\right\rangle \\
& =\frac{1}{2}\left(\left\|z_{n}-p\right\|^{2}+\left\|x_{n}-p\right\|^{2}-\left\|z_{n}-x_{n}\right\|^{2}\right)
\end{aligned}
$$

and hence

$$
\left\|z_{n}-p\right\|^{2} \leq\left\|x_{n}-p\right\|^{2}-\left\|z_{n}-x_{n}\right\|^{2}
$$

Note that the following equality holds:

$$
\|t x+(1-t) y\|^{2}=t\|x\|^{2}+(1-t)\|y\|^{2}-t(1-t)\|x-y\|^{2}
$$

for all $t \in[0,1]$ and $x, y \in H$. So, from (1.6) and (3.18), we get

$$
\begin{aligned}
\left\|y_{n}-p\right\|^{2}= & \left\|\left(1-\gamma_{n}\right)\left(x_{n}-p\right)+\gamma_{n}\left(T_{\mu_{n}} W_{n} P_{C}\left(I-\delta_{n} B\right) z_{n}-p\right)\right\|^{2} \\
\leq & \gamma_{n}\left\|T_{\mu_{n}} W_{n} P_{C}\left(I-\delta_{n} B\right) z_{n}-p\right\|^{2}+\left(1-\gamma_{n}\right)\left\|x_{n}-p\right\|^{2} \\
& \quad-\gamma_{n}\left(1-\gamma_{n}\right)\left\|T_{\mu_{n}} W_{n} P_{C}\left(I-\delta_{n} B\right) z_{n}-x_{n}\right\|^{2} \\
= & \gamma_{n}\left\|z_{n}-p\right\|^{2}+\left(1-\gamma_{n}\right)\left\|x_{n}-p\right\|^{2}-\left(1-\gamma_{n}\right)\left\|y_{n}-x_{n}\right\|^{2} \\
\leq & \gamma_{n}\left(\left\|x_{n}-p\right\|^{2}-\left\|z_{n}-x_{n}\right\|^{2}\right)+\left(1-\gamma_{n}\right)\left\|x_{n}-p\right\|^{2} \\
= & \left\|x_{n}-p\right\|^{2}-\gamma_{n}\left\|z_{n}-x_{n}\right\|^{2} .
\end{aligned}
$$

Therefore, from Lemma 2.5, Lemma 3.1 and (3.19), we have

$$
\begin{aligned}
& \left\|x_{n+1}-p\right\|^{2} \\
& \quad=\left\|\alpha_{n}\left(\gamma f\left(W_{n} x_{n}\right)-A p\right)+\beta_{n}\left(x_{n}-T_{\mu_{n}} W_{n} y_{n}\right)+\left(I-\alpha_{n} A\right)\left(T_{\mu_{n}} W_{n} y_{n}-p\right)\right\|^{2}
\end{aligned}
$$




$$
\begin{aligned}
\leq & \left\|\beta_{n}\left(x_{n}-T_{\mu_{n}} W_{n} y_{n}\right)+\left(I-\alpha_{n} A\right)\left(T_{\mu_{n}} W_{n} y_{n}-p\right)\right\|^{2} \\
& +2 \alpha_{n}\left\langle\gamma f\left(W_{n} x_{n}\right)-A p, x_{n+1}-p\right\rangle \\
\leq & \left(\left\|I-\alpha_{n} A\right\|\left\|y_{n}-p\right\|+\beta_{n}\left\|x_{n}-T_{\mu_{n}} W_{n} y_{n}\right\|\right)^{2} \\
& +2 \alpha_{n}\left\|\gamma f\left(W_{n} x_{n}\right)-A p\right\|\left\|x_{n+1}-p\right\| \\
\leq & \left(\left(1-\alpha_{n} \bar{\gamma}\right)\left\|y_{n}-p\right\|+\beta_{n}\left\|x_{n}-T_{\mu_{n}} W_{n} y_{n}\right\|\right)^{2} \\
& +2 \alpha_{n}\left\|\gamma f\left(W_{n} x_{n}\right)-A p\right\|\left\|x_{n+1}-p\right\| \\
\leq & \left(1-\alpha_{n} \bar{\gamma}\right)^{2}\left\|y_{n}-p\right\|^{2}+\beta_{n}^{2}\left\|x_{n}-T_{\mu_{n}} W_{n} y_{n}\right\|^{2} \\
& +2\left(1-\alpha_{n} \bar{\gamma}\right) \beta_{n}\left\|y_{n}-p\right\|\left\|x_{n}-T_{\mu_{n}} W_{n} y_{n}\right\| \\
& +2 \alpha_{n}\left\|\gamma f\left(W_{n} x_{n}\right)-A p\right\|\left\|x_{n+1}-p\right\| \\
\leq & \left(1-\alpha_{n} \bar{\gamma}\right)^{2}\left(\left\|x_{n}-p\right\|^{2}-\gamma\left\|z_{n}-x_{n}\right\|^{2}\right)+\beta_{n}^{2}\left\|x_{n}-T_{\mu_{n}} W_{n} y_{n}\right\|^{2} \\
& +2\left(1-\alpha_{n} \bar{\gamma}\right) \beta_{n}\left\|y_{n}-p\right\|\left\|x_{n}-T_{\mu_{n}} W_{n} y_{n}\right\| \\
& +2 \alpha_{n}\left\|\gamma f\left(W_{n} x_{n}\right)-A p\right\|\left\|x_{n+1}-p\right\| .
\end{aligned}
$$

Then we derive

$$
\begin{aligned}
&\left(1-\alpha_{n} \bar{\gamma}\right)^{2} \gamma_{n}\left\|z_{n}-x_{n}\right\|^{2} \\
& \leq\left\|x_{n}-p\right\|^{2}-\left\|x_{n+1}-p\right\|^{2}+\alpha_{n}^{2} \bar{\gamma}^{2}\left\|x_{n}-p\right\|^{2}+\beta_{n}^{2}\left\|x_{n}-T_{\mu_{n}} W_{n} y_{n}\right\|^{2} \\
&+2\left(1-\alpha_{n} \bar{\gamma}\right) \beta_{n}\left\|y_{n}-p\right\|\left\|x_{n}-T_{\mu_{n}} W_{n} y_{n}\right\| \\
&+2 \alpha_{n}\left\|\gamma f\left(W_{n} x_{n}\right)-A p\right\|\left\|x_{n+1}-p\right\| \\
& \leq\left(\left\|x_{n}-p\right\|+\left\|x_{n+1}-p\right\|\right)\left\|x_{n+1}-x_{n}\right\|+\alpha_{n}^{2} \bar{\gamma}^{2}\left\|x_{n}-p\right\|^{2} \\
&+\beta_{n}^{2}\left\|x_{n}-T_{\mu_{n}} W_{n} y_{n}\right\|^{2}+2\left(1-\alpha_{n} \bar{\gamma}\right) \beta_{n}\left\|y_{n}-p\right\|\left\|x_{n}-T_{\mu_{n}} W_{n} y_{n}\right\| \\
&+2 \alpha_{n}\left\|\gamma f\left(W_{n} x_{n}\right)-A p\right\|\left\|x_{n+1}-p\right\| .
\end{aligned}
$$

So, from (C4), Lemma 3.6, Lemma 3.8 and (3.20), we obtain

$$
\lim _{n \rightarrow \infty}\left\|x_{n}-z_{n}\right\|=0
$$

Lemma 3.10 $\lim _{n \rightarrow \infty}\left\|y_{n}-v_{n}\right\|=\lim _{n \rightarrow \infty}\left\|z_{n}-v_{n}\right\|=0$, where $v_{n}=P_{C}\left(I-\delta_{n} B\right) z_{n}$ for all $n \geq 0$.

Proof Let $p \in \mathcal{F}$. Setting $v_{n}=P_{C}\left(I-\delta_{n} B\right) z_{n}$ for all $n \geq 0$, since $p \in V I(C, B)$, we have $p=$ $P_{C}\left(I-\delta_{n} B\right) p$. From the $L$-Lipschitzian and relaxed $(\xi, v)$-cocoercive mapping on $B$ and Lemma 3.3, we have

$$
\begin{aligned}
& \left\|v_{n}-p\right\|^{2} \\
& \quad=\left\|P_{C}\left(I-\delta_{n} B\right) z_{n}-P_{C}\left(I-\delta_{n} B\right) p\right\|^{2} \\
& \quad \leq\left\|z_{n}-p-\delta_{n}\left(B z_{n}-B p\right)\right\|^{2} \\
& \quad=\left\|z_{n}-p\right\|^{2}-2 \delta_{n}\left\langle B z_{n}-B p, z_{n}-p\right\rangle+\delta_{n}^{2}\left\|B z_{n}-B p\right\|^{2}
\end{aligned}
$$


From (1.6) and (3.21), we get

$$
\begin{aligned}
\left\|y_{n}-p\right\|^{2} & =\left\|\left(1-\gamma_{n}\right) x_{n}+\gamma_{n} T_{\mu_{n}} W_{n} v_{n}-p\right\|^{2} \\
& =\left\|\left(1-\gamma_{n}\right)\left(x_{n}-p\right)+\gamma_{n}\left(T_{\mu_{n}} W_{n} v_{n}-p\right)\right\|^{2} \\
& \leq\left(1-\gamma_{n}\right)\left\|x_{n}-p\right\|^{2}+\gamma_{n}\left\|v_{n}-p\right\|^{2} \\
& \leq\left\|x_{n}-p\right\|^{2}+\gamma_{n}\left(2 \delta_{n} \xi+\delta_{n}^{2}-\frac{2 \delta_{n} v}{L^{2}}\right)\left\|B z_{n}-B p\right\|^{2} .
\end{aligned}
$$

From (1.6), Lemma 3.1 and (3.22), we have

$$
\begin{aligned}
\| x_{n+1}- & p \|^{2} \\
= & \| \alpha_{n}\left(\gamma f\left(W_{n} x_{n}\right)-A p\right)+\beta_{n}\left(x_{n}-p\right) \\
& +\left(\left(1-\beta_{n}\right) I-\alpha_{n} A\right)\left(T_{\mu_{n}} W_{n} y_{n}-p\right) \|^{2} \\
\leq & \left(\alpha_{n}\left\|\gamma f\left(W_{n} x_{n}\right)-A p\right\|+\beta_{n}\left\|x_{n}-p\right\|+\left(1-\beta_{n}-\alpha_{n} \bar{\gamma}\right)\left\|y_{n}-p\right\|^{2}\right. \\
= & \alpha_{n}^{2}\left\|\gamma f\left(W_{n} x_{n}\right)-A p\right\|^{2}+\beta_{n}^{2}\left\|x_{n}-p\right\|^{2}+\left(1-\beta_{n}-\alpha_{n} \bar{\gamma}\right)^{2}\left\|y_{n}-p\right\|^{2} \\
& +2 \alpha_{n}\left(\beta_{n}\left\|\gamma f\left(W_{n} x_{n}\right)-A p\right\|\left\|x_{n}-p\right\|\right. \\
& \left.+\left(1-\beta_{n}-\alpha_{n} \bar{\gamma}\right)\left\|\gamma f\left(W_{n} x_{n}\right)-A p\right\|\left\|y_{n}-p\right\|^{2}\right) \\
& +2 \beta_{n}\left(1-\beta_{n}-\alpha_{n} \bar{\gamma}\right)\left\|x_{n}-p\right\|\left\|y_{n}-p\right\| \\
\leq & \alpha_{n} M+\beta_{n}^{2}\left\|x_{n}-p\right\|^{2}+\left(1-\beta_{n}-\alpha_{n} \bar{\gamma}\right)^{2}\left\|y_{n}-p\right\| \\
& +\beta_{n}\left(1-\beta_{n}-\alpha_{n} \bar{\gamma}\right)\left(\left\|x_{n}-p\right\|^{2}+\left\|y_{n}-p\right\|^{2}\right) \\
= & \alpha_{n} M+\beta_{n}\left(1-\alpha_{n} \bar{\gamma}\right)\left\|x_{n}-p\right\|^{2}+\left(1-\alpha_{n} \bar{\gamma}\right)\left(1-\beta_{n}-\alpha_{n} \bar{\gamma}\right)\left\|y_{n}-p\right\|^{2} \\
\leq & \alpha_{n} M+\beta_{n}\left(1-\alpha_{n} \bar{\gamma}\right)\left\|x_{n}-p\right\|^{2}+\left(1-\alpha_{n} \bar{\gamma}\right)\left(1-\beta_{n}-\alpha_{n} \bar{\gamma}\right) \\
& \times\left(\left\|x_{n}-p\right\|^{2}+\gamma_{n}\left(2 \delta_{n} \xi+\delta_{n}^{2}-\frac{2 \delta_{n} v}{L^{2}}\right)\left\|B z_{n}-B p\right\|^{2}\right) \\
\leq & \alpha_{n} M+\left(1-\alpha_{n} \bar{\gamma}\right)^{2}\left\|x_{n}-p\right\|^{2} \\
& +\left(1-\alpha_{n} \bar{\gamma}\right)^{2} \gamma_{n}\left(2 \delta_{n} \xi+\delta_{n}^{2}-\frac{2 \delta_{n} v}{L^{2}}\right)\left\|B z_{n}-B p\right\|^{2} \\
= & \alpha_{n} M+\left\|x_{n}-p\right\|^{2} \\
& +\left(1-\alpha_{n} \bar{\gamma}\right)^{2} \gamma_{n}\left(2 \delta_{n} \xi+\delta_{n}^{2}-\frac{2 \delta_{n} v}{L^{2}}\right)\left\|B z_{n}-B p\right\|^{2} . \\
&
\end{aligned}
$$

It follows that

$$
\begin{aligned}
0 & \leq-\left(1-\alpha_{n} \bar{\gamma}\right)^{2} \gamma_{n}\left(2 \delta_{n} \xi+\delta_{n}^{2}-\frac{2 \delta_{n} \nu}{L^{2}}\right)\left\|B z_{n}-B p\right\|^{2} \\
& \leq \alpha_{n} M+\left\|x_{n}-p\right\|^{2}-\left\|x_{n+1}-p\right\|^{2}
\end{aligned}
$$




$$
\leq \alpha_{n} M+\left(\left\|x_{n}-p\right\|+\left\|x_{n+1}-p\right\|\right)\left\|x_{n+1}-x_{n}\right\|
$$

$\longrightarrow 0$,

which implies that

$$
\lim _{n \rightarrow \infty}\left\|B z_{n}-B p\right\|=0
$$

On the other hand, since $P_{C}$ is firmly nonexpansive, by Lemma 3.2, we have

$$
\begin{aligned}
\left\|v_{n}-p\right\|^{2}= & \left\|P_{C}\left(I-\delta_{n} B\right) z_{n}-P_{C}\left(I-\delta_{n} B\right) p\right\|^{2} \\
\leq & \left\langle\left(I-\delta_{n} B\right) z_{n}-\left(I-\delta_{n} B\right) p, v_{n}-p\right\rangle \\
= & \frac{1}{2}\left\{\left\|\left(I-\delta_{n} B\right) z_{n}-\left(I-\delta_{n} B\right) p\right\|^{2}+\left\|v_{n}-p\right\|^{2}-\left\|z_{n}-v_{n}-\gamma_{n}\left(B z_{n}-B p\right)\right\|^{2}\right\} \\
\leq & \frac{1}{2}\left\{\left\|z_{n}-p\right\|^{2}+\left\|v_{n}-p\right\|^{2}-\left\|z_{n}-v_{n}\right\|^{2}\right. \\
& \left.+2 \gamma_{n}\left\|z_{n}-v_{n}\right\|\left\|B z_{n}-B p\right\|-\gamma_{n}^{2}\left\|B z_{n}-B p\right\|^{2}\right\},
\end{aligned}
$$

which yields that

$$
\begin{aligned}
\left\|v_{n}-p\right\|^{2} & \leq\left\|z_{n}-p\right\|^{2}-\left\|z_{n}-v_{n}\right\|^{2}+2 \gamma_{n}\left\|z_{n}-v_{n}\right\|\left\|B z_{n}-B p\right\| \\
& \leq\left\|x_{n}-p\right\|^{2}-\left\|z_{n}-v_{n}\right\|^{2}+2 \gamma_{n}\left\|z_{n}-v_{n}\right\|\left\|B z_{n}-B p\right\|
\end{aligned}
$$

Combining (3.22) and (3.25), we obtain

$$
\begin{aligned}
\left\|y_{n}-p\right\|^{2} \leq & \left(1-\gamma_{n}\right)\left\|x_{n}-p\right\|^{2}+\gamma_{n}\left\|v_{n}-p\right\|^{2} \\
\leq & \left(1-\gamma_{n}\right)\left\|x_{n}-p\right\|^{2}+\gamma_{n}\left(\left\|x_{n}-p\right\|^{2}-\left\|z_{n}-v_{n}\right\|^{2}\right. \\
& \left.+2 \gamma_{n}\left\|z_{n}-v_{n}\right\|\left\|B z_{n}-B p\right\|\right) \\
= & \left\|x_{n}-p\right\|^{2}-\gamma_{n}\left\|z_{n}-v_{n}\right\|^{2}+2 \gamma_{n}\left\|z_{n}-v_{n}\right\|\left\|B z_{n}-B p\right\| .
\end{aligned}
$$

Therefore, from (3.23) and (3.26), we get

$$
\begin{aligned}
\left\|x_{n+1}-p\right\|^{2} \leq & \alpha_{n} M+\beta_{n}\left(1-\alpha_{n} \bar{\gamma}\right)\left\|x_{n}-p\right\|^{2}+\left(1-\alpha_{n} \bar{\gamma}\right)\left(1-\beta_{n}-\alpha_{n} \bar{\gamma}\right)\left\|y_{n}-p\right\|^{2} \\
\leq & \alpha_{n} M+\beta_{n}\left(1-\alpha_{n} \bar{\gamma}\right)\left\|x_{n}-p\right\|^{2}+\left(1-\alpha_{n} \bar{\gamma}\right)\left(1-\beta_{n}-\alpha_{n} \bar{\gamma}\right) \\
& \quad \times\left(\left\|x_{n}-p\right\|^{2}-\gamma\left\|z_{n}-v_{n}\right\|^{2}+2 \gamma_{n}^{2}\left\|z_{n}-v_{n}\right\|\left\|B z_{n}-B p\right\|\right) \\
\leq & \alpha_{n} M+\left(1-\alpha_{n} \bar{\gamma}\right)^{2}\left\|x_{n}-p\right\|^{2}-\left(1-\alpha_{n} \bar{\gamma}\right)\left(1-\beta_{n}-\alpha_{n} \bar{\gamma}\right) \gamma_{n}\left\|z_{n}-v_{n}\right\|^{2} \\
& +2\left(1-\alpha_{n} \bar{\gamma}\right)\left(1-\beta_{n}-\alpha_{n} \bar{\gamma}\right) \gamma_{n}^{2}\left\|z_{n}-v_{n}\right\|\left\|B z_{n}-B p\right\| .
\end{aligned}
$$

Hence, we have

$$
\begin{aligned}
& \left(1-\alpha_{n} \bar{\gamma}\right)\left(1-\beta_{n}-\alpha_{n} \bar{\gamma}\right) \gamma_{n}\left\|z_{n}-v_{n}\right\|^{2} \\
& \leq \alpha_{n} M+\left(\left\|x_{n}-p\right\|+\left\|x_{n+1}-p\right\|\right)\left\|x_{n+1}-x_{n}\right\| \\
& \quad+2\left(1-\alpha_{n} \bar{\gamma}\right)\left(1-\beta_{n}-\alpha_{n} \bar{\gamma}\right) \gamma_{n}^{2}\left\|z_{n}-v_{n}\right\|\left\|B z_{n}-B p\right\|,
\end{aligned}
$$


which implies that

$$
\lim _{n \rightarrow \infty}\left\|z_{n}-v_{n}\right\|=0 .
$$

Observe that

$$
\begin{aligned}
\left\|y_{n}-v_{n}\right\| \leq & \left\|y_{n}-x_{n}\right\|+\left\|x_{n}-z_{n}\right\|+\left\|z_{n}-v_{n}\right\| \\
\leq & \gamma_{n}\left\|T_{\mu_{n}} W_{n} v_{n}-x_{n}\right\|+\left\|x_{n}-z_{n}\right\|+\left\|z_{n}-v_{n}\right\| \\
\leq & \gamma_{n}\left(\left\|T_{\mu_{n}} W_{n} v_{n}-T_{\mu_{n}} W_{n} y_{n}\right\|+\left\|T_{\mu_{n}} W_{n} y_{n}-x_{n}\right\|\right) \\
& +\left\|x_{n}-z_{n}\right\|+\left\|z_{n}-v_{n}\right\| \\
\leq & \gamma_{n}\left(\left\|v_{n}-y_{n}\right\|+\left\|T_{\mu_{n}} W_{n} y_{n}-x_{n}\right\|\right)+\left\|x_{n}-z_{n}\right\|+\left\|z_{n}-v_{n}\right\|,
\end{aligned}
$$

and hence

$$
\left(1-\gamma_{n}\right)\left\|y_{n}-v_{n}\right\| \leq \gamma_{n}\left\|T_{\mu_{n}} W_{n} y_{n}-x_{n}\right\|+\left\|x_{n}-z_{n}\right\|+\left\|z_{n}-v_{n}\right\| .
$$

Thus, from Lemma 3.8, Lemma 3.9, (3.27) and (C4), we derive

$$
\lim _{n \rightarrow \infty}\left\|y_{n}-v_{n}\right\|=0 \text {. }
$$

Lemma 3.11 $P_{\mathcal{F}}(\gamma f+(I-A))$ is a contraction of $H$ into itself.

Proof From Lemma 3.1, we have

$$
\begin{aligned}
& \left\|P_{\mathcal{F}}(\gamma f+(I-A)) x-P_{\mathcal{F}}(\gamma f+(I-A)) y\right\| \\
& \quad \leq\|\gamma f(x)+(I-A) x-\gamma f(y)-(I-A) y\| \\
& \quad \leq \gamma\|f(x)-f(y)\|+\|(I-A)(x-y)\| \\
& \quad \leq \gamma \alpha\|x-y\|+(1-\bar{\gamma})\|x-y\| \\
& \quad=(1-(\bar{\gamma}-\gamma \alpha))\|x-y\|,
\end{aligned}
$$

for all $x, y \in H$. From the condition $\bar{\gamma}, 0<\alpha \gamma<\bar{\gamma}<\alpha \gamma+1$, we obtain $1-(\bar{\gamma}-\gamma \alpha) \in(0,1)$. Therefore, $P_{\mathcal{F}}(\gamma f+(I-A))$ is a contraction.

Now, we prove Theorem 3.1.

Proof of Theorem 3.1 From Lemma 3.11 and the Banach contraction principle, $P_{\mathcal{F}}(\gamma f+$ $(I-A))$ has a unique fixed point, say $x^{*} \in H$. That is, $x^{*}=P_{\mathcal{F}}(\gamma f+(I-A)) x^{*}$. Then, using (2.1), $x^{*}$ is the unique solution of the variational inequality

$$
\left\langle(\gamma f-A) x^{*}, x-x^{*}\right\rangle \leq 0
$$

for all $x \in \mathcal{F}$. Now, we show that

$$
\limsup _{n \rightarrow \infty}\left\langle(\gamma f-A) x^{*}, x_{n}-x^{*}\right\rangle \leq 0 \text {. }
$$


To show this, we can choose a subsequence $\left\{x_{n_{i}}\right\}$ of $\left\{x_{n}\right\}$ such that

$$
\limsup _{n \rightarrow \infty}\left\langle(\gamma f-A) x^{*}, x_{n}-x^{*}\right\rangle=\lim _{i \rightarrow \infty}\left\langle(\gamma f-A) x^{*}, x_{n_{i}}-x^{*}\right\rangle .
$$

Since $\left\{x_{n_{i}}\right\}$ is bounded, there exists a subsequence $\left\{x_{n_{i_{j}}}\right\}$ of $\left\{x_{n_{i}}\right\}$ which converges weakly to $z$. Without loss of generality, we can assume that $x_{n_{i}} \rightarrow z$. We need to show that $z \in \mathcal{F}=$ $F(\mathfrak{s}) \cap \Omega \cap\left(\bigcap_{n=1}^{\infty} F\left(T_{n}\right)\right) \cap V I(C, B)$.

(I) Since $x_{n_{i}} \rightarrow z$, by Lemma 2.2 and Lemma 3.7, we get

$$
T_{t} z=z
$$

for all $t \in S$. Therefore, $z \in F(\Im)$.

(II) Now, we show that $z \in \Omega$. Since $z_{n}=S_{r_{n}} x_{n}$, we derive

$$
\theta\left(z_{n}, x\right)+\varphi(x)-\varphi\left(z_{n}\right)+\frac{1}{r_{n}}\left\langle K^{\prime}\left(z_{n}\right)-K^{\prime}\left(x_{n}\right), \eta\left(x, z_{n}\right)\right\rangle \geq 0,
$$

for all $x \in C$. From the monotonicity of $\theta$, we have

$$
\theta\left(x, z_{n}\right) \leq-\theta\left(z_{n}, x\right) \leq \varphi(x)-\varphi\left(z_{n}\right)+\frac{1}{r_{n}}\left\langle K^{\prime}\left(z_{n}\right)-K^{\prime}\left(x_{n}\right), \eta\left(x, z_{n}\right)\right\rangle
$$

and hence

$$
\theta\left(x, z_{n_{i}}\right) \leq \varphi(x)-\varphi\left(z_{n_{i}}\right)+\left\langle\frac{K^{\prime}\left(z_{n_{i}}\right)-K^{\prime}\left(x_{n_{i}}\right)}{r_{n_{i}}}, \eta\left(x, z_{n_{i}}\right)\right\rangle .
$$

Since $\frac{K^{\prime}\left(z_{n_{i}}\right)-K^{\prime}\left(x_{n_{i}}\right)}{r_{n_{i}}} \rightarrow 0$ and $z_{n_{i}} \rightarrow z$, from the lower semicontinuity of $\varphi$ and (E4), we have

$$
\theta(x, z)+\varphi(z)-\varphi(x) \leq 0
$$

for all $x \in C$. For $t$ with $0<t \leq 1$ and $x \in C$, let $x_{t}=t x+(1-t) z$. Since $x \in C$ and $z \in C$, we have $x_{t} \in C$ and

$$
\theta\left(x_{t}, z\right)+\varphi(z)-\varphi\left(x_{t}\right) \leq 0 \text {. }
$$

From (E1), (E4) and the convexity of $\varphi$, we get

$$
\begin{aligned}
0 & =\theta\left(x_{t}, x_{t}\right)+\varphi\left(x_{t}\right)-\varphi\left(x_{t}\right) \\
& \leq t \theta\left(x_{t}, x\right)+(1-t) \theta\left(x_{t}, z\right)+t \varphi(x)+(1-t) \varphi(z)-\varphi\left(x_{t}\right) \\
& \leq t\left(\theta\left(x_{t}, x\right)+\varphi(x)-\varphi\left(x_{t}\right)\right) .
\end{aligned}
$$

Hence,

$$
\theta\left(x_{t}, x\right)+\varphi(x)-\varphi\left(x_{t}\right) \geq 0,
$$


for all $x \in C$. From (E3) and the lower semicontinuity of $\varphi$, we have

$$
\theta(z, x)+\varphi(x)-\varphi(z) \geq 0
$$

for all $x \in C$. Therefore, $z \in \Omega$.

(III) We show that $z \in F(W)=\bigcap_{i=1}^{\infty} F\left(T_{i}\right)$. Assume that $z \notin F(W)$, then $z \neq W z$. Since $z \in F(\Im) \cap \Omega$, by our assumption, we have $T_{i} z \in F(\Im), \forall i \in \mathbb{N}$ and then $W_{n} z \in F(\Im)$. From Lemma 2.1, we get

$$
T_{\mu_{n}} W_{n} z=W_{n} z
$$

for all $n \in \mathbb{N}$. Since

$$
\left\|y_{n}-x_{n}\right\| \leq\left\|y_{n}-v_{n}\right\|+\left\|v_{n}-z_{n}\right\|+\left\|z_{n}-x_{n}\right\|,
$$

from Lemma 3.9 and Lemma 3.10, we get

$$
\lim _{n \rightarrow \infty}\left\|y_{n}-x_{n}\right\|=0
$$

By Lemma 2.4, Lemma 3.8, (3.31) and (3.32), we obtain

$$
\begin{aligned}
& \liminf _{i \rightarrow \infty}\left\|x_{n_{i}}-z\right\| \\
& \quad<\liminf _{i \rightarrow \infty}\left\|x_{n_{i}}-W z\right\| \\
& \leq \liminf _{i \rightarrow \infty}\left(\left\|x_{n_{i}}-T_{\mu_{n_{i}}} W_{n_{i}} y_{n_{i}}\right\|+\left\|T_{\mu_{n_{i}}} W_{n_{i}} y_{n_{i}}-T_{\mu_{n_{i}}} W_{n_{i}} x_{n_{i}}\right\|\right. \\
& \left.\quad+\left\|T_{\mu_{n_{i}}} W_{n_{i}} x_{n_{i}}-T_{\mu_{n_{i}}} W_{n_{i}} z\right\|+\left\|T_{\mu_{n_{i}}} W_{n_{i}} z-W z\right\|\right) \\
& \leq \liminf _{i \rightarrow \infty}\left(\left\|y_{n_{i}}-x_{n_{i}}\right\|+\left\|x_{n_{i}}-z\right\|\right) \\
& \leq \liminf _{i \rightarrow \infty}\left\|x_{n_{i}}-z\right\| .
\end{aligned}
$$

This is a contraction. Therefore, $z \in F(W)=\bigcap_{i=1}^{\infty} F\left(T_{i}\right)$.

(IV) We show that $z \in V I(C, B)$. Let $U: H \rightarrow 2^{H}$ be a set-valued mapping defined by

$$
U x= \begin{cases}B x+N_{C} x, & \text { if } x \in C, \\ \emptyset, & \text { if } x \notin C,\end{cases}
$$

where $N_{C} x$ is the normal cone to $C$ at $x \in C$. By assumption of $B$, we have

$$
\begin{aligned}
\langle B x-B y, x-y\rangle & \geq-\xi\|B x-B y\|^{2}+v\|x-y\|^{2} \\
& \geq\left(v-\xi L^{2}\right)\|x-y\|^{2} \\
& \geq 0,
\end{aligned}
$$


which implies that $B$ is monotone. Thus, $U$ is a maximal monotone. Let $(u, v) \in G(U)$. Since $v-B u \in N_{C} u$ and $v_{n}=P_{C}\left(I-\delta_{n} B\right) z_{n} \in C$, we have

$$
\left\langle u-v_{n}, v-B u\right\rangle \geq 0 .
$$

On the other hand, from (2.1), we have

$$
\left\langle u-v_{n}, v_{n}-\left(I-\delta_{n} B\right) z_{n}\right\rangle \geq 0
$$

and hence

$$
\left\langle u-v_{n}, \frac{v_{n}-z_{n}}{\delta_{n}}+B z_{n}\right\rangle \geq 0 .
$$

It follows by (3.33) and (3.34) that

$$
\begin{aligned}
\left\langle u-v_{n_{i}}, v\right\rangle \geq & \left\langle u-v_{n_{i}}, B u\right\rangle \\
\geq & \left\langle u-v_{n_{i}}, B u\right\rangle-\left\langle u-v_{n_{i}}, \frac{v_{n_{i}}-z_{n_{i}}}{\delta_{n_{i}}}+B z_{n_{i}}\right\rangle \\
= & \left\langle u-v_{n_{i}}, B u-B z_{n_{i}}-\frac{v_{n_{i}}-z_{n_{i}}}{\delta_{n_{i}}}\right\rangle \\
= & \left\langle u-v_{n_{i}}, B u-B v_{n_{i}}\right\rangle+\left\langle u-v_{n_{i}}, B v_{n_{i}}-B z_{n_{i}}\right\rangle \\
& -\left\langle u-v_{n_{i}}, \frac{v_{n_{i}}-z_{n_{i}}}{\delta_{n_{i}}}\right\rangle \\
\geq & \left\langle u-v_{n_{i}}, B v_{n_{i}}-B z_{n_{i}}\right\rangle-\left\langle u-v_{n_{i}}, \frac{v_{n_{i}}-z_{n_{i}}}{\delta_{n_{i}}}\right\rangle .
\end{aligned}
$$

From Lemma 3.9 and Lemma 3.10, we obtain $\langle u-z, v\rangle \geq 0$ as $i \rightarrow \infty$. Since $U$ is maximal monotone, we have $z \in U^{-1}(0)$. Therefore, $z \in V I(C, B)$. By (I)-(IV), $z \in \mathcal{F}=F(\Im) \cap \Omega \cap$ $\left(\bigcap_{n=1}^{\infty} F\left(T_{n}\right)\right) \cap V I(C, B)$. Since $x^{*}=P_{\mathcal{F}}(\gamma f+(I-A)) x^{*}$, from (3.28), we have

$$
\begin{aligned}
\limsup _{n \rightarrow \infty}\left\langle(\gamma f-A) x^{*}, x_{n}-x^{*}\right\rangle & =\limsup _{i \rightarrow \infty}\left\langle(\gamma f-A) x^{*}, x_{n_{i}}-x^{*}\right\rangle \\
& =\limsup _{i \rightarrow \infty}\left\langle(\gamma f-A) x^{*}, z_{n_{i}}-x^{*}\right\rangle \\
& =\left\langle(\gamma f-A) x^{\prime \prime}, z-x^{*}\right\rangle \\
& \leq 0 .
\end{aligned}
$$

(V) Finally, we prove that $\left\{x_{n}\right\},\left\{y_{n}\right\}$ and $\left\{z_{n}\right\}$ converge strongly to $x^{*}$. From (1.6), we obtain

$$
\begin{aligned}
\left\|x_{n+1}-x^{*}\right\|^{2}= & \| \alpha_{n}\left(\gamma f\left(W_{n} x_{n}\right)-A x^{*}\right)+\beta_{n}\left(x_{n}-x^{*}\right) \\
& +\left(\left(1-\beta_{n}\right) I-\alpha_{n} A\right)\left(T_{\mu_{n}} W_{n} y_{n}-x^{*}\right) \|^{2} \\
\leq & \left\|\beta_{n}\left(x_{n}-x^{\prime \prime}\right)+\left(\left(1-\beta_{n}\right) I-\alpha_{n} A\right)\left(T_{\mu_{n}} W_{n} y_{n}-x^{*}\right)\right\|^{2} \\
& +2 \alpha_{n}\left(\gamma f\left(W_{n} x_{n}\right)-A x^{*}, x_{n+1}-x^{*}\right\rangle \\
\leq & \left(\beta_{n}\left\|x_{n}-x^{*}\right\|+\left\|\left(\left(1-\beta_{n}\right) I-\alpha_{n} A\right)\left(T_{\mu_{n}} W_{n} y_{n}-x^{*}\right)\right\|\right)^{2}
\end{aligned}
$$




$$
\begin{aligned}
& +2 \alpha_{n} \gamma\left\langle f\left(W_{n} x_{n}\right)-f\left(x^{*}\right), x_{n+1}-x^{*}\right\rangle \\
& +2 \alpha_{n}\left\langle\gamma f\left(x^{*}\right)-A x^{*}, x_{n+1}-x^{*}\right\rangle \\
\leq & \left(\beta_{n}\left\|x_{n}-x^{*}\right\|+\left(1-\beta_{n}-\alpha_{n} \bar{\gamma}\right)\left\|y_{n}-x^{*}\right\|\right)^{2} \\
& +2 \alpha_{n} \gamma \alpha\left\|x_{n}-x^{*}\right\|\left\|x_{n+1}-x^{*}\right\|+2 \alpha_{n}\left\langle\gamma f\left(x^{*}\right)-A x^{*}, x_{n+1}-x^{*}\right\rangle \\
\leq & \left(1-\alpha_{n} \bar{\gamma}\right)^{2}\left\|x_{n}-x^{*}\right\|^{2}+\alpha_{n} \gamma \alpha\left(\left\|x_{n}-x^{*}\right\|^{2}+\left\|x_{n+1}-x^{*}\right\|^{2}\right) \\
& +2 \alpha_{n}\left\langle\gamma f\left(x^{*}\right)-A x^{*}, x_{n+1}-x^{*}\right\rangle,
\end{aligned}
$$

which implies that

$$
\begin{aligned}
\left\|x_{n+1}-x^{*}\right\|^{2} & \\
\leq & \frac{1-2 \alpha_{n} \bar{\gamma}+\left(\alpha_{n} \bar{\gamma}\right)^{2}+\alpha_{n} \gamma \alpha}{1-\alpha_{n} \gamma \alpha}\left\|x_{n}-x^{*}\right\|^{2} \\
& +\frac{2 \alpha_{n}}{1-\alpha_{n} \gamma \alpha}\left\langle\gamma f\left(x^{*}\right)-A x^{*}, x_{n+1}-x^{*}\right\rangle \\
= & \left(1-\frac{2(\bar{\gamma}-\gamma \alpha) \alpha_{n}}{1-\alpha_{n} \gamma \alpha}\right)\left\|x_{n}-x^{* \prime}\right\|^{2}+\frac{\left(\alpha_{n} \bar{\gamma}\right)^{2}}{1-\alpha_{n} \gamma \alpha}\left\|x_{n}-x^{*}\right\|^{2} \\
& +\frac{2 \alpha_{n}}{1-\alpha_{n} \gamma \alpha}\left\langle\gamma f\left(x^{*}\right)-A x^{*}, x_{n+1}-x^{*}\right\rangle \\
= & \left(1-\frac{2(\bar{\gamma}-\gamma \alpha) \alpha_{n}}{1-\alpha_{n} \gamma \alpha}\right)\left\|x_{n}-x^{* \prime}\right\|^{2} \\
& +\frac{2(\bar{\gamma}-\gamma \alpha) \alpha_{n}}{1-\alpha_{n} \gamma \alpha}\left(\frac{\alpha_{n} \bar{\gamma}^{2}}{2(\bar{\gamma}-\gamma \alpha)}\left\|x_{n}-x^{*}\right\|^{2}+\frac{1}{\bar{\gamma}-\gamma \alpha}\left\langle\gamma f\left(x^{*}\right)-A x^{*}, x_{n+1}-x^{*}\right\rangle\right) .
\end{aligned}
$$

It follows that

$$
\left\|x_{n+1}-x^{*}\right\|^{2} \leq\left(1-b_{n}\right)\left\|x_{n}-x^{*}\right\|+b_{n} c_{n},
$$

where

$$
\begin{aligned}
& b_{n}=\frac{2(\bar{\gamma}-\gamma \alpha) \alpha_{n}}{1-\alpha_{n} \gamma \alpha}, \\
& c_{n}=\frac{\alpha_{n} \bar{\gamma}^{2}}{2(\bar{\gamma}-\gamma \alpha)}\left\|x_{n}-x^{\prime \prime}\right\|^{2}+\frac{1}{\bar{\gamma}-\gamma \alpha}\left\langle\gamma f\left(x^{*}\right)-A x^{*}, x_{n+1}-x^{\prime \prime}\right\rangle .
\end{aligned}
$$

From (C4)-(i), we have $\sum_{n=0}^{\infty} b_{n}=\infty$, and by (3.29), we get $\limsup _{n \rightarrow \infty} c_{n} \leq 0$. Consequently, applying Lemma 2.10 to (3.35), we get $\left\|x_{n}-x^{*}\right\| \rightarrow 0$. Therefore,

$$
\lim _{n \rightarrow \infty} x_{n}=x^{*}
$$

From Lemma 3.9 and (3.32), we obtain

$$
\lim _{n \rightarrow \infty} y_{n}=x^{*} \quad \text { and } \quad \lim _{n \rightarrow \infty} z_{n}=x^{*} .
$$

This completes the proof of Theorem 3.1. 
Corollary 3.1 Let $H, C, S, \Im, X,\left\{\mu_{n}\right\}, \varphi, \theta, T_{i}, f, A,\left\{\alpha_{n}\right\},\left\{\beta_{n}\right\}$ and $\left\{\gamma_{n}\right\}$ be as in Theorem 3.1. Suppose that $\mathcal{F}=\bigcap_{n=1}^{\infty} F\left(T_{n}\right) \cap F(\Im) \cap \Omega \neq \emptyset$. Assume that

(C1) $\eta: C \times C \rightarrow H$ is Lipschitz continuous with constant $\lambda>0$ such that

(a) $\eta(x, y)+\eta(y, x)=0, \forall x, y \in C$,

(b) for each fixed $y \in C, x \mapsto \eta(y, x)$ is sequentially continuous from the weak topology to the weak topology;

(C2) $K: C \rightarrow \mathbb{R}$ is $\eta$-strongly convex with constant $\sigma>0$ and its derivative $K^{\prime}$ is not only sequentially continuous from the weak topology to the strong topology, but also Lipschitz continuous with constant $v>0$ such that $\sigma \geq \lambda \nu$;

(C3) for each $x \in C$, there exist a bounded subset $D_{x} \subset C$ and $z_{x} \in C$ such that for any $y \in C \backslash D_{x}$,

$$
\theta\left(y, z_{x}\right)+\varphi\left(z_{x}\right)-\varphi(y)+\frac{1}{r}\left\langle K^{\prime}(y)-K^{\prime}(x), \eta\left(z_{x}, y\right)\right\rangle<0
$$

(C4) (i) $\lim _{n \rightarrow \infty} \alpha_{n}=0, \sum_{n=0}^{\infty} \alpha_{n}=\infty$,

(ii) $0<\liminf _{n \rightarrow \infty} \beta_{n} \leq \limsup _{n \rightarrow \infty} \beta_{n}<1$,

$0<\liminf _{n \rightarrow \infty} \gamma_{n} \leq \limsup _{n \rightarrow \infty} \gamma_{n}<1$,

(iii) $\lim _{n \rightarrow \infty}\left|\gamma_{n+1}-\gamma_{n}\right|=0$;

(C5) $\liminf _{n \rightarrow \infty} r_{n}>0$ and $\lim _{n \rightarrow \infty}\left|r_{n+1}-r_{n}\right|=0$.

Given $x_{0} \in C$ is arbitrary, let the sequences $\left\{x_{n}\right\},\left\{y_{n}\right\}$ and $\left\{z_{n}\right\}$ be generated by

$$
\left\{\begin{array}{l}
\theta\left(z_{n}, x\right)+\varphi(x)-\varphi\left(z_{n}\right)+\frac{1}{r_{n}}\left\langle K^{\prime}\left(z_{n}\right)-K^{\prime}\left(x_{n}\right), \eta\left(x, z_{n}\right)\right\rangle \geq 0, \\
y_{n}=\left(1-\gamma_{n}\right) x_{n}+\gamma_{n} T_{\mu_{n}} W_{n} P_{C} z_{n}, \\
x_{n+1}=\alpha_{n} \gamma f\left(W_{n} x_{n}\right)+\beta_{n} x_{n}+\left(\left(1-\beta_{n}\right) I-\alpha_{n} A\right) T_{\mu_{n}} W_{n} y_{n}, \quad n \geq 0 .
\end{array}\right.
$$

Then $\left\{x_{n}\right\},\left\{y_{n}\right\}$ and $\left\{z_{n}\right\}$ converge strongly to $x^{*} \in \mathcal{F}$, where $x^{*}=P_{\mathcal{F}}(\gamma f+(I-A)) x^{*}$, which solves the following variational inequality:

$$
\left\langle(\gamma f-A) x^{*}, x-x^{*}\right\rangle \leq 0, \quad \forall x \in \mathcal{F} .
$$

Proof Setting $B=0$ in Theorem 3.1, we obtain the required result.

Corollary 3.2 Let $H, C, S, \Im, X,\left\{\mu_{n}\right\}, T_{i}, f, A,\left\{\alpha_{n}\right\},\left\{\beta_{n}\right\}$ and $\left\{\gamma_{n}\right\}$ be as in Theorem 3.1. Suppose that $\mathcal{F}=\bigcap_{n=1}^{\infty} F\left(T_{n}\right) \cap F(\Im) \neq \emptyset$. Assume that

(C1) (i) $\lim _{n \rightarrow \infty} \alpha_{n}=0, \sum_{n=0}^{\infty} \alpha_{n}=\infty$,

(ii) $0<\liminf _{n \rightarrow \infty} \beta_{n} \leq \limsup _{n \rightarrow \infty} \beta_{n}<1$, $0<\liminf _{n \rightarrow \infty} \gamma_{n} \leq \limsup _{n \rightarrow \infty} \gamma_{n}<1$,

(iii) $\lim _{n \rightarrow \infty}\left|\gamma_{n+1}-\gamma_{n}\right|=0$;

(C2) $\liminf _{n \rightarrow \infty} r_{n}>0$ and $\lim _{n \rightarrow \infty}\left|r_{n+1}-r_{n}\right|=0$.

Given $x_{0} \in C$ is arbitrary, let the sequences $\left\{x_{n}\right\},\left\{y_{n}\right\}$ and $\left\{z_{n}\right\}$ be generated by

$$
\left\{\begin{array}{l}
y_{n}=\left(1-\gamma_{n}\right) x_{n}+\gamma_{n} T_{\mu_{n}} W_{n} P_{C} x_{n}, \\
x_{n+1}=\alpha_{n} \gamma f\left(W_{n} x_{n}\right)+\beta_{n} x_{n}+\left(\left(1-\beta_{n}\right) I-\alpha_{n} A\right) T_{\mu_{n}} W_{n} y_{n}, \quad n \geq 0 .
\end{array}\right.
$$


Then $\left\{x_{n}\right\},\left\{y_{n}\right\}$ and $\left\{z_{n}\right\}$ converge strongly to $x^{*} \in \mathcal{F}$, where $x^{*}=P_{\mathcal{F}}(\gamma f+(I-A)) x^{*}$, which solves the following variational inequality:

$$
\left\langle(\gamma f-A) x^{*}, x-x^{*}\right\rangle \leq 0, \quad \forall x \in \mathcal{F} \text {. }
$$

Proof Set $B=0, \theta(x, y)=0$ for all $x, y \in C, \varphi=0$ and $r_{n}=1$ for all $n \geq 1$. Take $K(x)=\frac{\|x\|^{2}}{2}$ and $\eta(x, y)=x-y$ for all $x, y \in C$. From (1.6), we have

$$
\left\{\begin{array}{l}
y_{n}=\left(1-\gamma_{n}\right) x_{n}+\gamma_{n} T_{\mu_{n}} W_{n} P_{C} x_{n}, \\
x_{n+1}=\alpha_{n} \gamma f\left(W_{n} x_{n}\right)+\beta_{n} x_{n}+\left(\left(1-\beta_{n}\right) I-\alpha_{n} A\right) T_{\mu_{n}} W_{n} y_{n}, \quad n \geq 0 .
\end{array}\right.
$$

Then the conclusion immediately follows from Theorem 3.1.

Corollary 3.3 Let $H, C, S, \Im, X,\left\{\mu_{n}\right\}, \varphi, \theta, f, A,\left\{\alpha_{n}\right\},\left\{\beta_{n}\right\}$ and $\left\{\gamma_{n}\right\}$ be as in Theorem 3.1. Suppose that $\Omega \neq \emptyset$. Assume that

(C1) $\eta: C \times C \rightarrow H$ is Lipschitz continuous with constant $\lambda>0$ such that

(a) $\eta(x, y)+\eta(y, x)=0, \forall x, y \in C$,

(b) for each fixed $y \in C, x \mapsto \eta(y, x)$ is sequentially continuous from the weak topology to the weak topology;

(C2) $K: C \rightarrow \mathbb{R}$ is $\eta$-strongly convex with constant $\sigma>0$, and its derivative $K^{\prime}$ is not only sequentially continuous from the weak topology to the strong topology, but also Lipschitz continuous with constant $v>0$ such that $\sigma \geq \lambda \nu$;

(C3) for each $x \in C$, there exist a bounded subset $D_{x} \subset C$ and $z_{x} \in C$ such that for any $y \in C \backslash D_{x}$,

$$
\theta\left(y, z_{x}\right)+\varphi\left(z_{x}\right)-\varphi(y)+\frac{1}{r}\left\langle K^{\prime}(y)-K^{\prime}(x), \eta\left(z_{x}, y\right)\right\rangle<0
$$

(C4) (i) $\lim _{n \rightarrow \infty} \alpha_{n}=0, \sum_{n=0}^{\infty} \alpha_{n}=\infty$,

(ii) $0<\liminf _{n \rightarrow \infty} \beta_{n} \leq \limsup _{n \rightarrow \infty} \beta_{n}<1$,

$$
0<\liminf _{n \rightarrow \infty} \gamma_{n} \leq \limsup _{n \rightarrow \infty} \gamma_{n}<1 \text {, }
$$

(iii) $\lim _{n \rightarrow \infty}\left|\gamma_{n+1}-\gamma_{n}\right|=0$;

(C5) $\liminf _{n \rightarrow \infty} r_{n}>0$ and $\lim _{n \rightarrow \infty}\left|r_{n+1}-r_{n}\right|=0$.

Given $x_{0} \in C$ is arbitrary, let the sequences $\left\{x_{n}\right\},\left\{y_{n}\right\}$ and $\left\{z_{n}\right\}$ be generated by

$$
\left\{\begin{array}{l}
\theta\left(z_{n}, x\right)+\varphi(x)-\varphi\left(z_{n}\right)+\frac{1}{r_{n}}\left\langle K^{\prime}\left(z_{n}\right)-K^{\prime}\left(x_{n}\right), \eta\left(x, z_{n}\right)\right\rangle \geq 0, \\
y_{n}=\left(1-\gamma_{n}\right) x_{n}+\gamma_{n} P_{C} z_{n}, \\
x_{n+1}=\alpha_{n} \gamma f\left(x_{n}\right)+\beta_{n} x_{n}+\left(\left(1-\beta_{n}\right) I-\alpha_{n} A\right) y_{n}, \quad n \geq 0 .
\end{array}\right.
$$

Then $\left\{x_{n}\right\},\left\{y_{n}\right\}$ and $\left\{z_{n}\right\}$ converge strongly to $x^{*} \in \Omega$, where $x^{*}=P_{\Omega}(\gamma f+(I-A)) x^{*}$, which solves the following variational inequality:

$$
\left\langle(\gamma f-A) x^{*}, x-x^{*}\right\rangle \leq 0, \quad \forall x \in \Omega
$$

Proof Set $B=0$ and $T_{i} x=x$ for all $i=1,2, \ldots$ in (1.6). Then $W_{n} x=x$ for all $x \in C$. The conclusion immediately follows from Theorem 3.1. 


\section{Competing interests}

The author declares that they have no competing interests.

\section{Acknowledgements}

The author would like to thank Prof. Anthony To-Ming Lau for his helpful suggestions and the referees for their valuable comments and suggestions which improved the presentation of this paper. This research was supported by Basic Science Research Program through the National Research Foundation of Korea (NRF) funded by the Ministry of Education, Science and Technology (2012R1A1A4A01010526).

\section{Received: 21 June 2012 Accepted: 5 October 2012 Published: 24 October 2012}

\section{References}

1. Moudafi, A: Viscosity approximation methods for fixed points problems. J. Math. Anal. Appl. 241, 46-55 (2000)

2. Xu, HK: Viscosity approximation methods for nonexpansive mappings. J. Math. Anal. Appl. 298, $279-291$ (2004)

3. Marino, G, Xu, HK: A general iterative method for nonexpansive mappings in Hilbert spaces. J. Math. Anal. Appl. 318, 43-52 (2006)

4. Rockafellar, RT: On the maximality of sums of nonlinear monotone operators. Trans. Am. Math. Soc. 149, 75-88 (1970)

5. liduka, $\mathrm{H}$, Takahashi, W: Strong convergence theorems for nonexpansive mappings and inverse strongly monotone mappings. Nonlinear Anal. 61(3), 341-350 (2005)

6. Takahashi, W: Weak and strong convergence theorems for families of nonexpansive mappings and their applications. Ann. Univ. Mariae Curie-Sk̄odowska 51, 277-292 (1997)

7. Takahashi, W, Shimoji, K: Convergence theorems for nonexpansive mappings and feasibility problems. Math. Comput. Model. 32, 1463-1471 (2000)

8. Yao, Y, Cho, YJ, Chen, R: An iterative algorithm for solving fixed point problems, variational inequality problems and mixed equilibrium problems. Nonlinear Anal. 71, 3363-3373 (2009)

9. Ceng, LC, Yao, JC: A hybrid iterative scheme for mixed equilibrium problems and fixed point problems. J. Comput. Appl. Math. 214(1), 186-201 (2008)

10. Chang, SS, Lee, HWJ, Chan, CK: Generalized system for relaxed cocoercive variational inequalities in Hilbert spaces. Appl. Math. Lett. 20, 329-334 (2007)

11. Kim, JK, Kim, KS: New systems of generalized mixed variational inequalities with nonlinear mappings in Hilbert spaces. J. Comput. Anal. Appl. 12(3), 601-612 (2010)

12. Jaiboon, C, Kumam, P: A general iterative method for addressing mixed equilibrium problems and optimization problems. Nonlinear Anal. 73(5), 1180-1202 (2010)

13. Kim, KS: Convergence of a hybrid algorithm for a reversible semigroup of nonlinear operators in Banach spaces Nonlinear Anal. 73, 3413-3419 (2010)

14. Saeidi, S: Iterative algorithms for finding common solutions of variational inequalities and systems of equilibrium problems and fixed points of families and semigroups of nonexpansive mappings. Nonlinear Anal. 70, 4195-4208 (2009)

15. Lau, AT-M: Invariant means on almost periodic functions and fixed point properties. Rocky Mt. J. Math. 3, 69-76 (1973)

16. Lau, AT-M: Invariant means and fixed point properties of semigroup of nonexpansive mappings. Taiwan. J. Math. 12(6), 1525-1542 (2008)

17. Lau, AT-M, Takahashi, W: Invariant means and fixed point properties for nonexpansive representations of topological semigroups. Topol. Methods Nonlinear Anal. 5, 39-57 (1995)

18. Day, MM: Amenable semigroup. III. J. Math. 1, 509-544 (1957)

19. Lau, AT-M, Shioji, N, Takahashi, W: Existence of nonexpansive retractions for amenable semigroups of nonexpansive mappings and nonlinear ergodic theorems in Banach spaces. J. Funct. Anal. 161, 62-75 (1999)

20. Holmes, RD, Lau, AT-M: Non-expansive actions of topological semigroups and fixed points. J. Lond. Math. Soc. 5(2), 330-336 (1972)

21. Lau, AT-M, Zhang, Y: Fixed point properties of semigroups of nonexpansive mappings. J. Funct. Anal. 254(10), 2534-2554 (2008)

22. Jitpeera, T, Kumam, P: A new iterative algorithm for solving common solutions of generalized mixed equilibrium problems, fixed point problems and variational inclusion problems with minimization problems. Fixed Point Theory Appl. 2012, $111(2012)$

23. Katchang, P, Kumam, P: An iterative algorithm for common fixed points for nonexpansive semigroups and strictly pseudo-contractive mappings with optimization problems. J. Glob. Optim. (2012). doi:10.1007/s10898-012-9927-y

24. Katchang, P, Kumam, P: A composite implicit iterative process with a viscosity method for Lipschitzian semigroup in a smooth Banach space. Bull. Iran. Math. Soc. 37(1), 143-159 (2011)

25. Katchang, P, Kumam, P: Hybrid-extragradient type methods for a generalized equilibrium problem and variational inequality problems of nonexpansive semigroups. Fixed Point Theory 13, 107-120 (2012)

26. Katchang, P, Kumam, P: A system of mixed equilibrium problems, a general system of variational inequality problems for relaxed cocoercive and fixed point problems for nonexpansive semigroup and strictly pseudo-contractive mappings. J. Appl. Math. 2012, Article ID 414831 (2012)

27. Kumam, $P$, Katchang, P: The hybrid algorithm for system of mixed equilibrium problems, general system of finite variational inequalities and common fixed points for nonexpansive semigroups and strictly pseudo-contractive mappings. Fixed Point Theory Appl. 2012, 84 (2012)

28. Onjai-uea, N, Katchang, P, Kumam, P: Strong convergence theorems for solving a general system of finite variational inequalities for finite accretive operators and fixed points of nonexpansive semigroups with weak contraction mappings. Fixed Point Theory Appl. 2012, 114 (2012)

29. Sunthrayuth, $P$, Kumam, P: Iterative algorithms approach to a general system of nonlinear variational inequalities with perturbed mappings and fixed point problems for nonexpansive semigroups. J. Inequal. Appl. 2012, 113 (2012)

30. Sunthrayuth, P, Kumam, P: An iterative method for solving a system of mixed equilibrium problems, system of quasivariational inclusions and fixed point problems of nonexpansive semigroups with application to optimization problems. Abstr. Appl. Anal. 2012, Article ID 979870 (2012) 
31. Kada, O, Lau, AT-M, Takahashi, W: Asymptotically invariant net and fixed point set for semigroup of nonexpansive mappings. Nonlinear Anal. 29(5), 539-550 (1997)

32. Takahashi, W: Nonlinear Functional Analysis: Fixed Point Theory and Applications. Yokohama Publishers, Yokohama (2000)

33. Hanson, MA: On sufficiency of the Kuhn-Tucker conditions. J. Math. Anal. Appl. 80, 545-550 (1981)

34. Ansari, QH, Yao, JC: Iterative scheme for solving mixed variational-like inequalities. J. Optim. Theory Appl. 108(3), 527-541 (2001)

35. Köthe, G: Topological Vector Spaces I. Springer, Berlin (1983)

36. Opial, Z: Weak convergence of the sequence of successive approximations for nonexpansive mappings. Bull. Am. Math. Soc. 73, 591-597 (1967)

37. Shimoji, K, Takahashi, W: Strong convergence to common fixed points of infinite nonexpansive mappings and applications. Taiwan. J. Math. 5, 387-404 (2001)

38. Ceng, LC, Yao, JC: Hybrid viscosity approximation scheme for equilibrium problems and fixed point problems of infinitely many nonexpansive mappings. Appl. Math. Comput. 198, 729-741 (2008)

39. Suzuki, T: Strong convergence of Krasnoselskii and Mann's type sequences for one-parameter nonexpansive semigroups without Bochner integrals. J. Math. Anal. Appl. 305(1), 227-239 (2005)

40. Bruck, RE: On the convex approximation property and the asymptotic behavior of non-linear contractions in Banach spaces. Isr. J. Math. 38, 304-314 (1981)

doi:10.1186/1687-1812-2012-185

Cite this article as: Kim: Convergence to common solutions of various problems for nonexpansive mappings in Hilbert spaces. Fixed Point Theory and Applications 2012 2012:185.

\section{Submit your manuscript to a SpringerOpen ${ }^{\circ}$ journal and benefit from:}

- Convenient online submission

- Rigorous peer review

- Immediate publication on acceptance

- Open access: articles freely available online

- High visibility within the field

- Retaining the copyright to your article 